\title{
ACNP 60th Annual Meeting: Keyword Index
}

\author{
Neuropsychopharmacology (2021) 46:554-569; https://doi.org/10.1038/s41386-021-01240-x
}

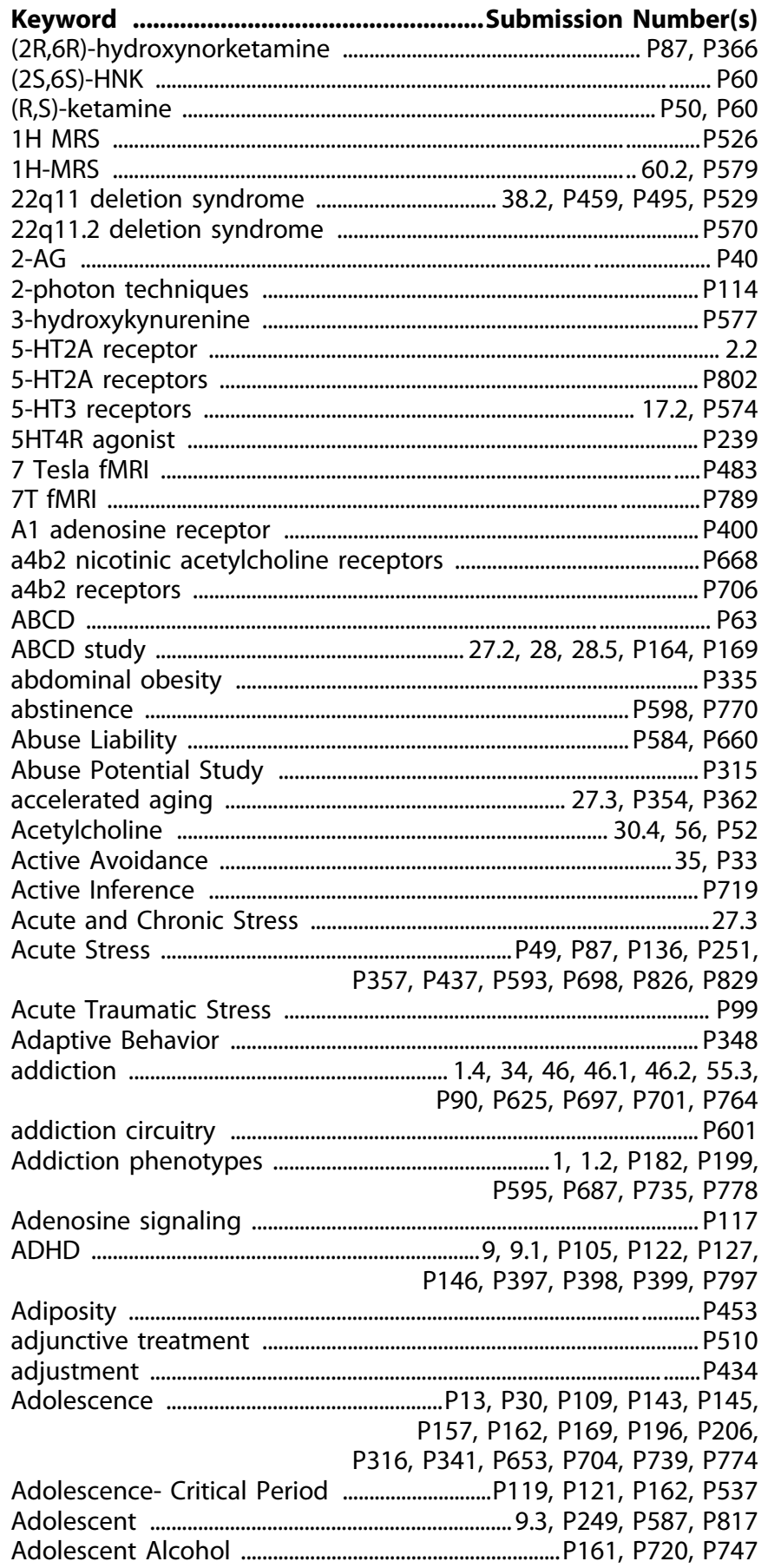

Adolescent Alcohol Use ................................................P691, P702, P774 Adolescent Anxiety .............................................................. P159, P763 Adolescent binge drinking ..................................................................P763 Adolescent Brain Cognitive Development Study .........................P801 Adolescent Depression ................................................... 43.4, P282, P325 adolescent development .....................................................................P148

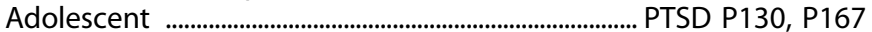
adolescent schizophrenia ...................................................................P160 adolescent stress ………………….........................................P578, P787

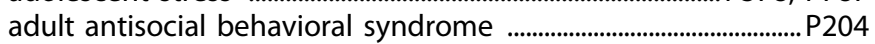
adult hippocampal neurogenesis .......................................P217, P259 Adverse childhood events .................................................... 14, P170 adverse childhood experiences (ACE) ...............................................P430

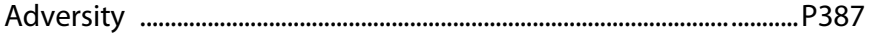
aerobic exercise ................................................................................... P108 affective behavior ...........................................................................42, 56.4 affective components of pain ............................................... 42.2, P339 affective neuroscience .......................................................................... P172

Affective theory of mind .................................................................... P143 African American ..............................................................................................2. 27.1 age effects ..................................................................................................P104

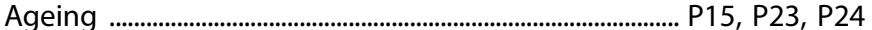

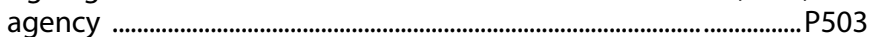
aggression ......................................... 39.2, 47, P127, P210, P238, P635 Aging …........................................................ 33, P4, P26, P530 Air Pollution $44,44.3,44.4$

Alcohol

$17.4,31.4,46.1,55.2$, 55.3, 56.4, P249, P263, P604, P635, P664, P666, P672, P685, P686, P692, P700, P704, P707, P726, P771, P777, P778

Alcohol and substance use disorders 17.3, 42.1, P205, P595, P611, P671, P703, P711, P769

Alcohol consumption 34.2, P332, P632,

P725, P778

Alcohol dependence P184, P618,

alcohol drinking P681, P698, P762

Alcohol epigenetic marks alcohol preference ........................................................................P750

Alcohol Sensitivity .................................................................................P332 alcohol use disorder .........................................31.2, P285, P605, P610, P618, P648, P649, P680, P678, P741, P766, P767

Alcohol Use Disorder - Treatment $17.1,17.2,34,34.2$ 34.3, P630, P639, P734 Alcohol use disorder and drug addiction ..................25.3, 45.4, 46.3, $\mathrm{P} 687, \mathrm{P} 704$ alcohol withdrawal ................................ P591, P644, P662, P683, P692

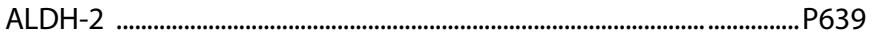
allopregnanolone .................................................................. 31, 31.3, 31.5 
allosteric modulator P330

alpha oscillations

alpha7 nicotinic acetylcholine receptor

$6,6.5$, P617

Altitude

P281, P338

Alzheimer's dementia

P791

Alzheimer's Disease .48, 56, 56.1, 56.2, 56.3, P2,

P6, P7, P8, P10, P16, P19,

P25, P306, P409, P782

Ambiguity

Amphetamine

.P826

Amygdala

P446, P505, P576, P597

29.4, 39.3, 43.3, 45.2, 46.1,

P27, P41, P84, P89, P95,

P236, P267, P337, P387, P447,

P653, P747, P759, P793, P794

amygdala-based networks

analgesia

43.4

Analgesic

P366, P408, P732

Anandamide

Androgen

..42.4

anhedonia

51.1, P128, P247,

P282, P310, P324,

P341, P388, P789, P803

Animal Models $25.3,46.2,57,57.2$

P338, P463, P661, P725, P730

$14,14.1,14.3$,

anorexia nervosa

P187, P189, P196

Antagonist Ligands P590

anterior cingulate 37.5

Anterior Cingulate Cortex (ACC)

$35,35.4,42.2,55.2$

P33, P175, P260, P331,

P339, P441, P528, P769

Anterior insula

35.2, P260, P578, P604

anterior limb of the internal capsule

anthranilic acid

35.5

anticholinergic

...P577

Anticholinergic medication burden

. P573

Antidepressant

P477, P573

21.4, P12, P218, P297,

P315, P369, P390, P400

Antidepressant Induced Mania

P302

antidepressant pharmacotherapy ............................................................ 8.4

Antidepressant Treatment .................................................................P394

Antidepressant Trials ..................................................................................... 8.2

Antidepressants ...................................................... 8.1, P183, P333, P448

anti-gliadin antibodies (AGA) .............................................................P569

Anti-inflammatory ...................................................................................... P366

anti-inflammatory cytokines ....................................................................34.3

Antipsychotic

P463, P493, P507, P547, P559

Antipsychotic agents

P453

Antipsychotic drugs

P510

Antipsychotic response .............................................................60, P506

Antipsychotic Treatment ........................................................ P17, P373

Antipsychotic-naïve first-episode schizophrenia ...............P506, P542

antipsychotic-naive schizophrenia ..................................................P526

Antipsychotics

P458, P509, P563, P577

antisocial personality disorder

Anxiety

9, 9.2, 22, 37.4, P7, P32, P37, P43,

$\mathrm{P} 54, \mathrm{P} 58, \mathrm{P} 78, \mathrm{P} 90, \mathrm{P} 222, \mathrm{P} 239$

$\mathrm{P} 433, \mathrm{P} 633, \mathrm{P} 747, \mathrm{P} 816, \mathrm{P} 828$

Anxiety \& PTSD

31, P42, P55, P64, P88, P89

Anxiety and Depression $\mathrm{P} 80, \mathrm{P} 177, \mathrm{P} 234$ P309, P356, P786

Anxiety and stress

P11, P39, P41, P86, P140

Anxiety circuitry 22.4, P69, P331

Anxiety Development ..................................................................................2.26.2

Anxiety Disorder .......................................................................................... P92

anxiety disorders ...................................................................................... P28

anxiolytics

P283

Apoptosis ...P558 appetite P357

Approach/Avoidance

P73, P745

aromatase

P656

arousal

46.4

Arterial Spin Labeling

P148

Artifical transcription factors

artificial intelligence

P723

associative learning

$3,3.1, P 127$

Astrocyte

$P 27, P 655, P 785$

astrocyte-derived exosomes (ADE)

P301, P637, P713, P813

astrocytes

P117, P358, P717, P754

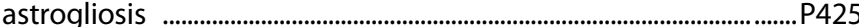

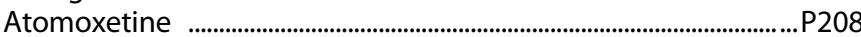

Attention ................................................................... P26, P208, P693

attentional bias ................................................................................... P203

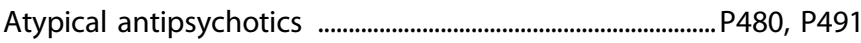

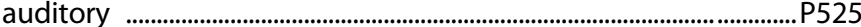

Auditory cortex ............................................................................................. P467

Auditory fear conditioning .......................................................... P33, P95

Auditory hallucinations .........................................................P467, P473

Auditory Mismatch Negativity ..................................P470, P562, P574

Auditory perception ...............................................................................P535

auditory processing ..................................................................................... P460

auditory steady-state response ............................................P562, P574

Auditory Striatum ................................................................................... P535

augmentation strategies $\ldots \ldots \ldots \ldots \ldots \ldots \ldots \ldots$

Autism …………………………………………..... 11, 16.2, 38.4, P124

$\mathrm{P} 129, \mathrm{P} 137, \mathrm{P} 142$

autism spectrum disorder

16.4, 36, P112, P120,

Autism spectrum disorder and

related syndromes

Automated Facial Affect Recognition

$16, \mathrm{P} 171, \mathrm{P} 512$

automated natural speech analysis

P495

avoidance

P292

avoidance and escape ............................................................................. P41

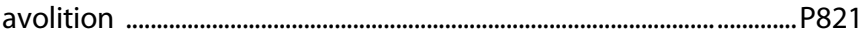

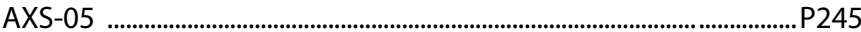

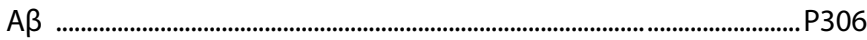

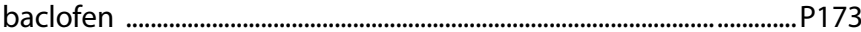

balloon analogue risk task ......................................................................... P695

Bariatric Surgery .......................................................................................... P197

Basal Ganglia .................................................................. 35.3, P413, P823

base excision DNA repair .................................................................... P381

basolateral amygdala .................................... 22.2, P36, P69, P665, P737

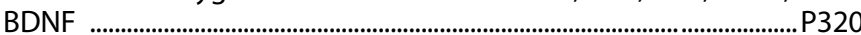

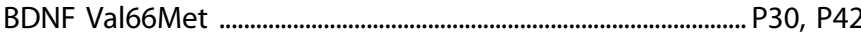

bed nucleus of the stria terminalis ....................................... 39.2, P825

behavior .......................................................................................................P570

behavior predictor .................................................................................... P105

behavioral activation system ...............................................................P200

behavioral economics ...........................................................................20.3

behavioral flexibility ...................................................... 35, 57, P591

behavioral genetics .................................................................................. P714

behavioral inhibition ............................................................................P313

behavioral inhibition system ................................................................P200

Behavioral Pharmacology ....................................................... 34.2, P627

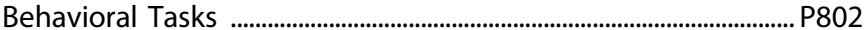

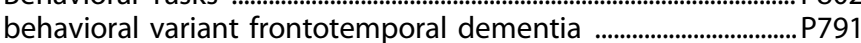

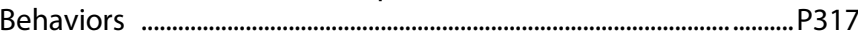

Biased signaling .............................................................................. 42.3, P600

big data .......................................................................................... P17

big data analysis .....................................................................30.2, 49

Binge alcohol use ........................................................................... P645

Binge eating .................................................................................. P188, P193

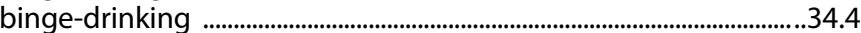

Binge-eating disorder ..................................................................................... P193 
Bioenergetics

P322, P338

bioinformatics

P355, P550, P611

Biomarker Fingerprint

Biomarker Prediction

P572

biomarkers

P140, P318

21, 31.5, 36, 60.4, P19, P29,

P163, P369, P375, P394, P496, P749

biostatistics ....................... 3

biotypes

bipolar depression

60.4

Bipolar Disorder

P305, P319, P373

9.1, 42.4, P17, P228, P236,

P238, P255, P270, P275, P284, P288,

P302, P304, P307, P321, P332, P362,

P363, P375, P381, P390, P397,

P398, P399, P504, P575

Bipolar I \& II disorder

P373

Bisque

P276

Blood pressure

P72

Blood-Brain-Barrier

4.2, P504, P530

BNST

body dysmorphic disorder

P86, P104, P290

Body mass index

P439

body perception

P186

body self

P187

BOLD fMRI signal

Borderline Personality Disorder

Brain

Brain age

Brain Anatomy

brain circuitry

brain connectome

Brain development

P149, P168, P292, P416

brain endophenotypes . . . . . . . . . . . . 7

brain imaging .................................................................. 9.1, 27, 55, P551

brain microvascular endothelial cells ........................P329, P504, P530

Brain Stimulation

$50.5,55$

Brain Structural Connectivity ................................................................. 565

brain structure

P675

Brain Transcription

39.5, P220

Brain-based Predictor

P799

Brain-enriched exosomes

... P38

Brainstem

56.1, P698

breast cancer chemotherapy

bulimia nervosa

P180

Buprenorphine

CA1

calcium imaging

$14,14.2,14.3$, P193

P486

callous/unemotional

P754, P823

P202

Callous-unemotional traits ............................................................ P143

CAMP signalling

P413

canabinoid receptors

Cannabidiol

Cannabinoids

cannabis

P424, P616, P742, P805

$P 26, P 620$

$13,13.2,13.3,42.4,55.4$,

P111, P481, P558, P606, P631, P633

cannabis use

P587, P615

cannabis use disorder

$13.5,55,55.5, \mathrm{P} 179$

P502, P583, P587, P693, P695, P749

Cardiolipin

..P297

Cardiometabolic Risk

P44, P226

cardiovascular

... P29

Cardiovascular Physiology

P53, P530

catalepsy

P732

catatonia

P533

causal discovery analyses

P150

CBT and antidepressants

21.3

Cell- and Circuit-selectivity

P177

Cell Type Specific
Cell-free DNA P375

Cell-type diversity

...P4

central amygdala

Central autonomic network

P681, P711

Central Nervous System

P326

Central Ner P509

c-Fos 28.5, P97, P321, P503

Chemobrain

chemogenetics

P437, P607, P722, P747

44.4, P114, P212, P277,

P594, P626, P676, P781, P808

chemotherapy-induced peripheral neuropathy ............................P420

Child abuse and neglect ....................................................................P110

child maltreatment ............................................................................P170

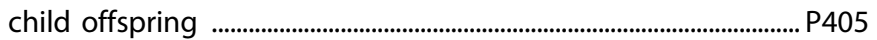

Childhood Adversity ............................24, 54.4, P44, P149, P296, P825

childhood anxiety ................................................................................................ P85

childhood maltreatment ........................................................................ P389

Childhood maltreatment exposure ....................................................P674

Childhood Onset Bipolar Disorder P288

Childhood trauma

14.4, P64, P110,

P130, P164, P748

Childhood-onset schizophrenia ..........................................................P160

Children

P111

Children and Adolescents

9, 54.4, P131

choice

P602

Choline analog ....u.u.u. P407

cholinergic interneuron

P207

cholinergic system

56.3, P4, P256, P407

CHRNA5

P686

chromatin ................................................................................................. P80

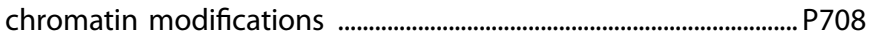

Chronic corticosterone

P340

chronic pain

P417, P608, P730

chronic social defeat

chronic stress

$P 221, P 290$

P212, P265, P300,

P301, P344, P395

Chronic unpredictable mild stress

cingulo-opercular network

P320, P340, P343

circadian

P409

circadian rhythm

P180, P383, P401, P532, P739

circRNA

Circuit Function in Humans ……………………………………......37.2

circuit optogenetics ........................................................................ 45, P814

Circuit-function ..............................................................................................54.1

Circuitry-based approach

37.2

circuits

39, P327, P672

Circulating cell-free mitochondrial DNA

28.3, P211, P255

Circulating mitochondrial DNA ...........................................................P275

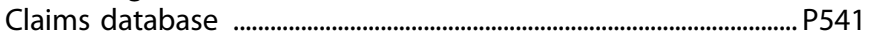

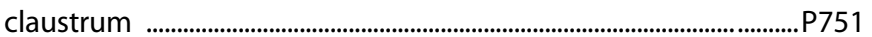

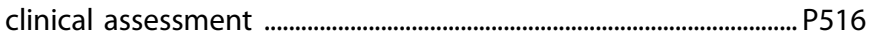

Clinical Development ............................................................................... 3.3

Clinical heterogeneity .................................................. 38.4, P226, P288

clinical high risk for psychosis ..................................................................... 36

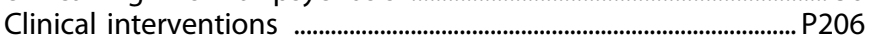

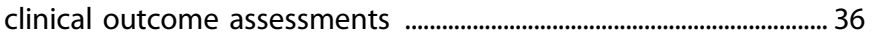

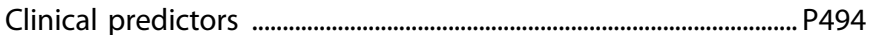

Clinical psychiatry ..................................................................................... P272

clinical psychopharmacology ……………………............................P507

Clinical trial

$13,37.3$, P28, P328,

Clinical Trial Rating Methods

P368, P386, P418, P630

clinical trials methodology

clozapine

P100

clozapine response

P160

Cluster Analysis

CNS Clinical Trials

60.4, P568, P579

3, 3.3, 36, P272,

P525, P554, P572 
coactivation pattern analysis cocaine

... 389

10.2, 29.3, P175, P596, P622, P636, P637, P640, P643, P656, P713, P751, P761, P764, P813 P609, P613, P657,

cocaine addiction P659, P752, P759, P772, P780

cocaine and opioid use disorders

cocaine seeking

cocaine self-administration

P693, P723

P770

P629, P714,

P740, P743, P756, P768

cocaine self-administration and reinstatement ................P607, P722

cocaine sex differences

$10,10.4$

cocaine use disorder

P583, P590, P671, P674,

P675, P740, P743, P804

cocaine-seeking behavior $\mathrm{P} 634, \mathrm{P} 740$

Cognition 1.4, 6.2, 13.2, 17.2, P5, P9, P13, P51, P374, P406, P455, P487, P539, P564, P572, P573, P617, P798, P813, P814

cognitive and affective neuroscience P670 cognitive behavioral therapy cognitive control

51.3

9.2, 18, 57, 57.4,

P143, P208, P209

cognitive control network 26.4, P804

Cognitive Decline P12, P664

cognitive development

Cognitive dysfunction

cognitive flexibility

P814, P827

$16.3,57.2, \mathrm{P} 324$

P346, P353, P710, P751

cognitive function .. P591

Cognitive Functioning ..P116, P477, P517, P537

Cognitive impairment associated

with schizophrenia ....

Cognitive impairments

P450, P462, P560, P562, P574

cognitive neuroscience

P499

Cognitive performance

...P196

cognitive skills

6.3, P538

.......P139

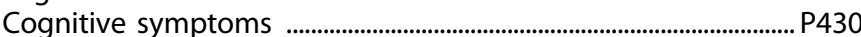

Cognitive training .......................................................................................P610

Commercial insurance .................................................................. P541

communication

5.58

Comorbidity

$17, \mathrm{P} 440$

Complement component ...................................................................... 452.4

complement pathway ............................................................................52.4

compulsion

$\mathrm{P} 604$

Compulsive behavior

$1,1.2,1.4,50.3,50.4$

compulsive drug intake

..P641

compulsivity

Computational Methods

$1.1,1.3, \mathrm{P} 194$

computational modeling

22.5, P461, P829

P433, P652, P719

computational models of decision-making ....................................P310

Computational Neuroscience .................................................... 3.2, P807

computational psychiatry ..................................................... 35.3, P324

Computational Reinforcement Learning Model .................. P55, P800

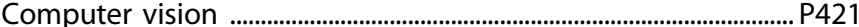

Computerized Cognitive Training .......................................................P576

COMT inhibitor ....................................................................................... P443

conditioned fear memory .................................................................... P69

conditioned place preference .........................P627, P642, P651, P656

conduct disorder ..................................................................................... P204

conflict monitoring ..................................................................................... 22.5

connectivity .................................................................................................... P22

Connectivity gradients ......................................................... P441

consortium .............................................................................................P736

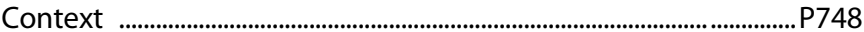

contextual fear ........................................................................................... P50

contingency management ..................................................................P179

Convulsion-like model ............................................................... P407

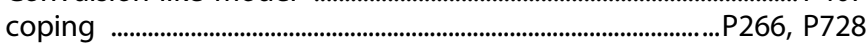

copy number variants .......................................................................... P512

corollary discharge ................................................................................ P503

Cortical atrophy …………........................................................................... P32

cortical circuit development ............................................................. P543

Cortical Development ........................................................................P138

cortical excitability ................................................................................P431

cortical excitation-inihbition balance ...............................................P232

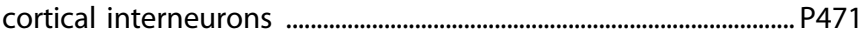

Cortical Myelination ................................................................................P107

cortical thickness ...................................................................................... P186

cortical thinning ............................................................................................... 52.4

corticolimbic ...................................................................................... 42, P766

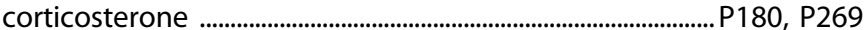

Corticosterone response to stress ....................................................P340

Corticostriatal circuit .............................................................................. P266

Cortico-striatal connectivity .................................................................P441

Corticotropin-releasing factor (CRF) ................................................. P252

Cortisol .......................................................................P58, P68, P251, P760

Cortisol response to stress .....................................................................P357

counting Stroop Functional MRI (fMRI) ............................................P203

Court-involved youth ...........................................................................P206

COVID-19 6, 6.2, 23, 23.2, 23.3, 23.4, 23.5, 54, P11, P99, P140, P151,

$\mathrm{P} 178, \mathrm{P} 183, \mathrm{P} 253, \mathrm{P} 272, \mathrm{P} 434$

P440, P544, P680, P700, P826

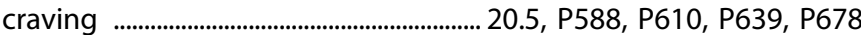

c-reactive protein ............................................................................... P498

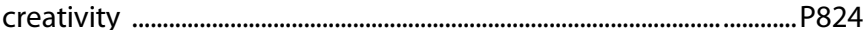

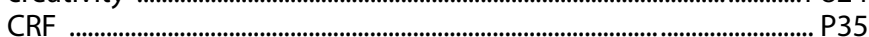

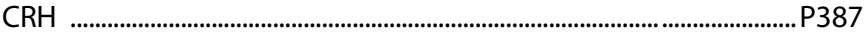

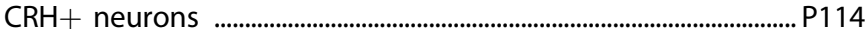

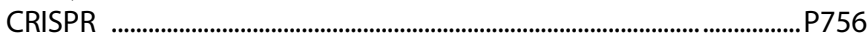

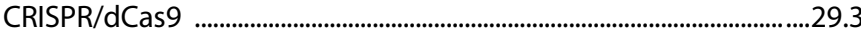

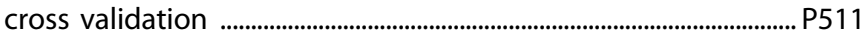

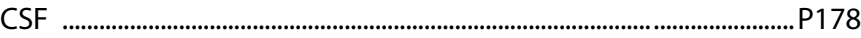

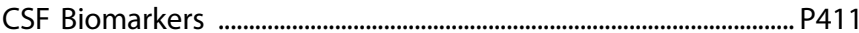

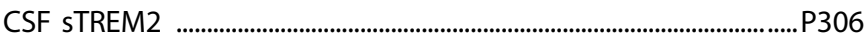

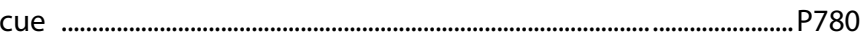

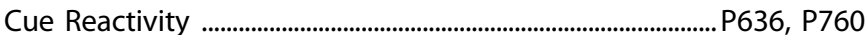

cue reinstatement ...................................................................................... P624

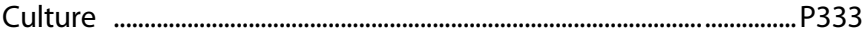

Cyberball ................................................................................................... P812

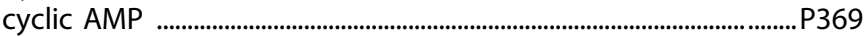

Cyclic peptide ........................................................................................P553

cytokines ............................................................... 52.3, P24, P38, P178

cytoskeleton ......................................................................................P536

D1 dopamine receptors ................................................. 57.5, P478, P535

D2 dopamine antagonists .................................................................. P507

D2 dopamine receptor ............................................... 22.2, P207, P534

D2 Receptor .................................................................................................P547

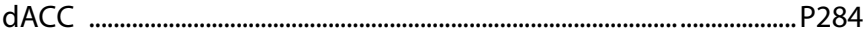

Dallas Heart Study ……………………………………………………………. P1

D-amino acid oxidase ...............................................................................P462

D-amino acid oxidase inhibitor ....................................................P560

daridorexant ....................................................................................................P582

DAT/NET inhibition ............................................................................. P619

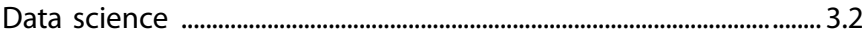

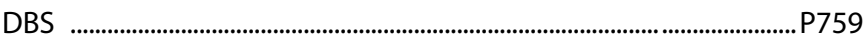

Decentralized Clinical Trials ......................................................................... 3.3

Decision Making ……………………………........ 22, 22.3, 22.5, 35.3, 46.4

P196, P410, P587, P622,

P635, P706, P787, P823, P826

declarative memory .....................................................................................P8

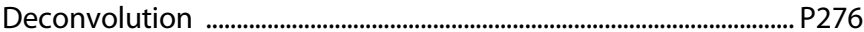

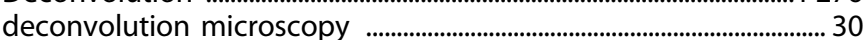

Default mode network (DMN) .................. 2.3, P74, P467, P475, P814

defensive and motivated behaviors ........................................... P89

delay discounting ............................................................................... 22.3, P198 
delta opioid receptor P665 delta9-tetrahydrocannabinol P589, P805 Dementia $37.3,48,48.2,48.4$

Dementia-related psychosis 48.3

Dendritic arborization dendritic morphogenesis

dendritic remodeling

Dendritic spines

dentate gyrus

Depression

...P795 .. P536

... P87, P598

P622, P663 ..P529

2.2, 2.5, 4, 4.2, 10.5, 16.3, $17.2,21,21.1,21.4,25.2,37.3,43$, 43.3, 46.4, 51, 51.1, 51.3, 56, 56.2,

P11, P20, P48, P97, P176, P211, $\mathrm{P} 221, \mathrm{P} 229, \mathrm{P} 230, \mathrm{P} 233, \mathrm{P} 238, \mathrm{P} 239$, P240, P243, P244, P247, P252, P261, P265, P268, P280, P291, P295, P299, P306, P316, P324, P327, P333, P336, P342, P343, P352, P368, P377, P390, P404, P406, P606, P762

Depression and Anxiety 10, 37.2, P109, P242, P338, P379, P382

depression and obesity P386 Depression Inflammation Cytokine ..4.4, P297, P361, P370 depression recurrence risk P261 Depression-CVD comorbidity ...25.4 depressive disorders depressive symptoms P169 development 51.2, P298, P359

Developmental 4.1, P640

Developmental Psychopathology

Developmental trajectory P4... P43

Developmental transcriptome P534

Diagnosis 11.1

Differential susceptibility

Diffusion Tensor Imaging (DTI)

..P575

P140

27.1, P57, P85,

P197, P376, P586, P752

Diffusion Weighted Imaging P487, P565, P605

Digital phenotyping

P214

digital psychiatry P228

Dimensional child psychopathology ......................................... 26.4, 28.5

dimensional psychopathology .......................................................... P85

dimensions ..........................................................................................................38.3

dimethyltryptamine ................................................................................ P365

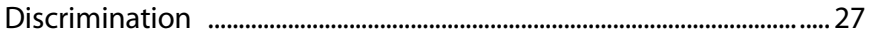

Disease risk prediction ...................................................................................... 3.1

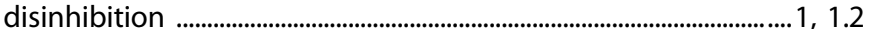

disruptive behavior disorders ................................................................. P156

diverse populations

diversity

DLPFC

$40,49, P 790$

.P567, P760

DNA

48.1, P566, P575

DNA hydroxymethylation

DNA Methylation 27, 31, P61, P227,

P362, P377, P479, P778

DNMT P755

Dopamine

6.2, 22.4, 29.3, 30.4, 39.3, 44.4, 51.1, P6, P47, P109, P129, P153, P165, P177, P191, P205, P268, P282, P330, P340, P388, P413, P427, P446, P535, P547, P597, P658, P679, P689, P691, P709, P710, P823 P699

Dopamine (D2, D3) receptors P642, P770 dopamine D3 receptors P590 Dopamine D4 Receptor P668 Dopamine function dopamine receptor type 2-expressing striatal medium spiny neuron

Dopamine receptors 45.3 dopamine transporter .42 .4

Dopaminergic system P650

dorsal caudate P165

Dorsal Hippocampus dorsal raphe

Dorsal Root Ganglia

Dorsal striatum

dorsal visual stream ..........P419 dorsolateral prefrontal cortex ................................ 6.5, 37.5,P484, P502 dorsolateral striatum ................................................................................. 10.4

Dorsomedial striatum .............................................................................5

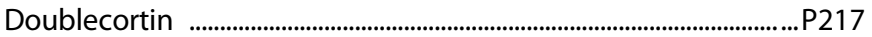

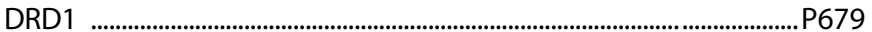

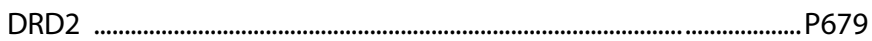

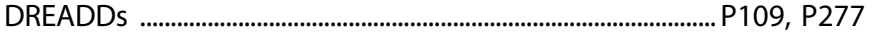

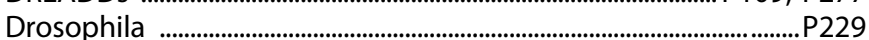

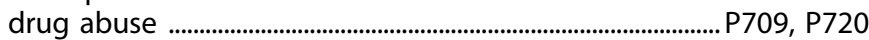

Drug Development ..............................................................P225, P547

Drug Discovery - new approaches ............................P355, P472, P563

drug discrimination ................................................................................. P584

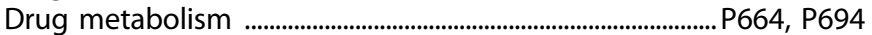

Drug Relapse .................................................................... 45.3, P657, P661

drug repurposing ............................................................................... P183

drug self-administration ..............................................34.4, 55.4, P623

D-serine ..................................................................... P462, P514, P525

Dual orexin receptor antagonist ......................................20.3, 20.4, 20.5,

P185, P581, P582,

P584, P764

duloxetine ....................................................................................................P367

dynamic causal modeling ....................................................................P260

Dynamic Functional Connectivity ....................................... P92, P353

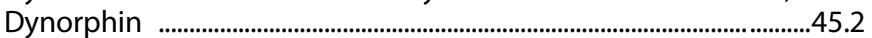

early adolescence .....................................................................................P471

early childhood .....................................................................................P135

Early identification of risk .................................................................P288

Early life adversity ...........................................................25.3, 27.3, 54.2

Early life stress ............................................... 10, 16.3, 25.2, 28, 29, 29.5,

44, 44.5, 54.1, 54.3, P44,

$\mathrm{P} 132, \mathrm{P} 134, \mathrm{P} 254, \mathrm{P} 294$,

P316, P675, P684, P700

early onset psychosis .. P512

early psychosis

P454, P474, P538,

early-life adversity

P555, P556, P565

10.3, 25.4, 29.5, 54,

early-life stress

eating behavior

P48, P430

Eating disorders P196

ecological momentary assessment

EEG

$14.4, \mathrm{P} 186$

EEG biomarkers

9, 9.1, 13.2, 21.4, P313, P522

EEG connectivity

9.2, 26.2, 57, 57.2, P314, P476

EEG electrophysiology

P522

EEG/ERP electrophysiology

....P436

fffectiveness

7.4, 57.4, P786

Efficacy and Tolerability

$8,8.3$

effort based decision making task

Effort Discounting

P122, P310, P498

ehmt2

electric field modeling

electroconvulsive therapy

electroencephalography

Electronic cigarette (e-cigarette)

electronic health record (EHR)

electrophysiology ..P324 P618 $P 232, P 323, P 810$ ..P289, P374, P406, P810 P552 P589, P660, P716

P472, P539, P702, P720, P751

EMA

embryo screening P540 
Emergency Department

P65, P788

emotion

P465

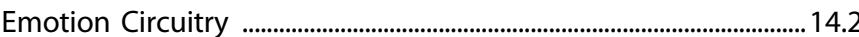

emotion processing ................................................................................P164

emotion regulation ............................................................................ P820

Emotional dysregulation

Emotional empathy

P528, P690

Emotional regulation

$14,25.3,43.4,53.2$

Emotional stress P780

emulated target trial

..P312

endocannabinoid system

$\mathrm{P} 59, \mathrm{P} 111$

Endocannabinoids

68, P696

endophenotypes

$38,38.5$

Endothelial function ............................................................................... P72

engram ........................................................................................................... P7

environment ........................................................................................... 162

environmental risk factors ................................................................ P37

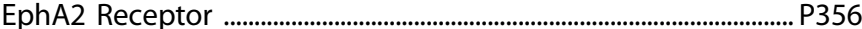

Ephrin .....................................................................................................P356

epidemiology ............................................ 48.2, P120, P133, P544, P550

epigenetic age acceleration .............................................................. P362

Epigenetic biomarkers

Epigenetic Modification

Epigenetics

25.1

$16.2, \mathrm{P} 75$

$2.2,10.3,16,16.4,16.5,25$

29, 29.2, 29.5, 31.2, P3, P36, P80,

P113, P294, P375, P479, P629, P664,

P708, P715, P744, P756, P778

epigenomics P269

epilepsy

$P 415, P 423$

equity

..... 40

ERP

Error processing ............................................................................. P436, P797

Escitalopram .................................................................................................P305

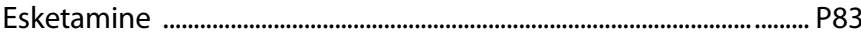

estradiol

10.4, 53.3, P327, P351

estrogen receptor

P327

estrogen therapy

.... 53

ethanol

P599, P644

ethanol intake

... P757

ethics

12,32

eugenics

...P540

event-related potentials ..............................9.3, P187, P470, P669, P811

evidence-based approach .................................................................P773

EWAS ……..........................................................................................P377

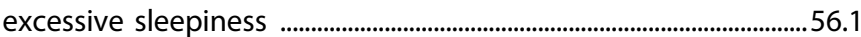

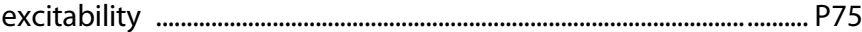

excitation-inhibition balance ..............................................................P471

executive function .................................................. 54.2, P8, P172, P195

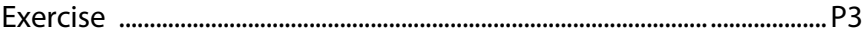

Experience dependent plasticity .........................................................54.1

experimental design ..................................................................................... 58

Experimental Therapeutics ........................................................ 47, P476

exploration ......................................................................................................P792

explore-exploit dilemma ........................................................ 719, P792

Exposure therapy …………………………………………............................ P54

extended amygdala ............................................................. P39, P825

Externalizing behavior ...............................................................................54.4

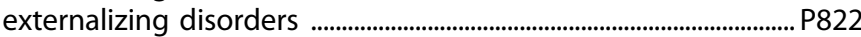

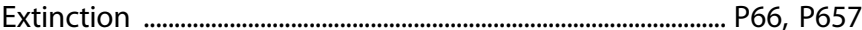

Extinction and Reinstatement .............................................................. P596

Extinction learning ............................................................................... P54

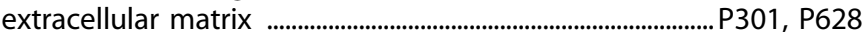

Extracellular vesicles ................................................................P248, P830

extrasynaptic GABA receptors ............................................................ P235

eye-blinks ............................................................................................................P679

F-18 PET Imaging .................................................................................. P58

Facial emotion processing factor analysis

P289

familial risk

familial risk of bipolar disorder

P363, P397, P398, P399

fast scan cyclic voltammetry

P288

Fast-acting Antidepressant

P650, P658

Fear

P311

Fear conditioning

Fear conditioning and extinction

P27, P41, P84, P239

P52, P60, P89, P130, P347

P31, P54, P55, P59,

P76, P209, P444

Fear extinction

P87, P92

fear generalization

Fear learning

P40

Fear physiology

56.4, P30, P75, P96

fear-potentiated startle ................................................................ 130, P185

Fecal microbiota transplant ................................................................P334

Feedback negativity ...............................................................................5.

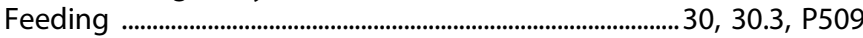

feeding behavior .................................................................. 11.3, P195

females ......................................................................................................P309

Fentanyl ....................................................................20, P598, P647, P718

fetal alcohol spectrum disorder ...........................................138, P664

Fetal Brain Development .........................................................................44.2

Fetal Origins .............................................................................................25.1

fiber photometry ................................... P136, P347, P446, P534, P676

fibromyalgia ..................................................................................................P176

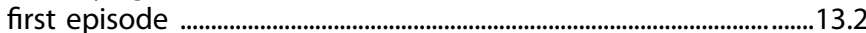

first episode psychosis ........................................................... 13.3, P455,

P474, P505, P515, P516, P531

first-episode schizophrenia ................................................................... P524

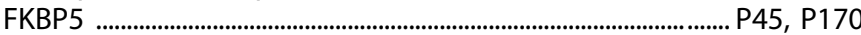

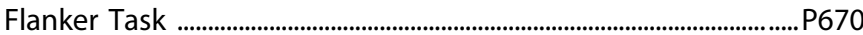

fluoxetine .......................................................................................... 50.2, P292

FMR1 premutation .............................................................................. P428

fMRI Biomarkers ........................................................................................... P304

fMRI Effective Connectivity ................................................................... P439

fMRI Functional Connectivity ....................................................P593

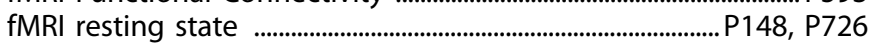

fNIRS ……….................................................................................. P631

focused ultrasound ........................................................2729, P793, P811

food craving ...................................................................................................... 335

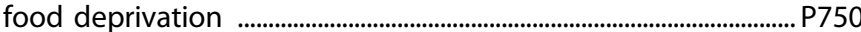

food intake ............................................................................................ P600

fragile $X$ syndrome .............................................................................P173

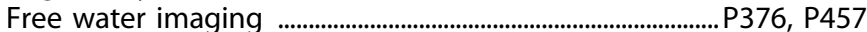

Frontolimbic network ..........................................................................P111

Frontoparietal Network ..................................................................P814

frontostriatal circuitry ............................................................................... P565

frontotemporal dementia ........................................................................... 48

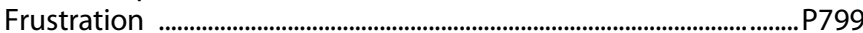

Frustrative Non-Reward ..................................................................47, P403

functional brain network ...................................................................P455

functional connectivity .................................................... P74, P112, P459,

$\mathrm{P} 469, \mathrm{P} 568, \mathrm{P} 825, \mathrm{P} 828$

functional disability

P319

Functional imaging

functional impairment

30.3

Functional MRI (fMRI)

P275, P298

$2.5,7,9.1,14.4,26,27.1$

37.2, 37.4, 51.1, 53, 55.3, 60.3,

P28, P59, P70, P76, P92, P137,

$\mathrm{P} 168, \mathrm{P} 193, \mathrm{P} 241, \mathrm{P} 243, \mathrm{P} 253$,

P277, P291, P307, P325, P326,

P353, P403, P418, P448, P551,

P570, P571, P625, P652, P690,

P769, P788, P797, P804, P822

functional near-infrared spectoscopy 26

functional neuroimaging

14.3, 53.3, P55

P444, P456 
functional outcomes

functional variants

P450

Functioning

fusiform face area (FFA)

P220

Future orientation

P359, P362

.P551

P119

G9a

P618

GABA

P163, P222, P223, P271,

GABA neuron P117, P300

GABAA

31.2

GABAA receptor positive allosteric modulator

GABA-A, positive allosteric modulators

P415

GABAergic interneurons

31.4, P235, P415

GABAergic system

P459, P464, P489

gambling

gambling disorder

P407

gaming disorder ...

$. .46,46.4$

P804

gamma oscillations

1.1

P552

GDF-15

P354

Gender differences

58

Gene co-expression networks

gene expression

..4.4, P96

10.2, 28.5, 51.4, P262,

P466, P482, P757

gene level analysis

P381

gene priming

..P708

generalized anxiety disorder

P32

genetic architecture .................................................................................. P445

Genetic Disorder .................................................................................... P105

genetic risk factor

.28 .4

Genetic variation

P199, P302

Genetically encoded sensor

$30.5, P 207$

genetics

Genetics of depression

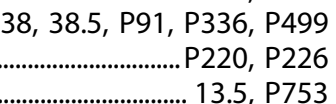

genome-wide association study

Genomics

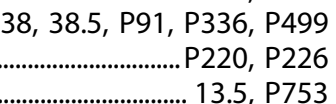

25, 25.4, 38.5, P94, P146,

P349, P566, P736, P798

geriatric

P17, P368

geriatric depression

8, P271

Ghrelin

P44, P251

Gliadin

P569

gliosis

P425

Globus Pallidus

P754

GLP-1 receptor

P726

GLT-1

P717

glucocorticoid

45, P49, P278

glucose metabolism

glutamate

... 22

glutamate GABA

60.2, P244, P254, P265,

P400, P427, P515, P813

Glutamate homeostasis

52.5, P404, P526

glutamate receptor ... P87

glutamate transporter (EAAT3)

glutamatergic synapses

P466

glutamatergic transmission

P343, P446, P619

P343, P591

glutathione

P567

gluten

P569

Glycocalyx

P5 542

glycosylation

P451

goal-directed behaviors

P754

Gonadal Hormones

.... 10

GoNoGo

P207

GPCR

P369, P417, P553, P563

GPS

P214

gray matter volumes

P147

GRIN1 mutation

P431

GRIN-related disorders

Gut Microbiome

$44,44,4,53,2, \mathrm{P} 20$

P129, P316, P647, P795
Gut-Brain Axis

P334, P740

GWAS

P184, P226, P694, P746

gyrification

P139

habenula

35.3, P380

habit

50.4, P754

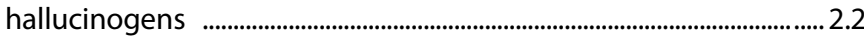

haplotype association mapping ………………………………........... P735

harm avoidance

Harm reduction ..........................................................................P616, P660

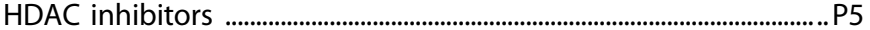

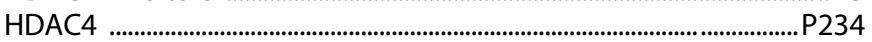

head-to-head clinical trial ....................................................................... 8.1

Health Disparities ..............................................................59, P19, P206

health policy ........................................................................................ 59

Health services …………………….................................................... P99

healthy individuals .................................................................................. P818

heart rate variability ..................................................53, 53.2, P126, P326

Hedonic Ingestion ................................................................................P190

hemodynamic effect ................................................................................... P286

Hemoglobin A1C

Heroin

.... P1

29.2, 45, 45.3, P615,

P650, P676, P717

heterogeneity

P298

high fat diet

P116, P192

High potency THC ...................................................................................... P616

Higher criticism ............................................................................................ P74

High-Throughput ........................................................................................ P829

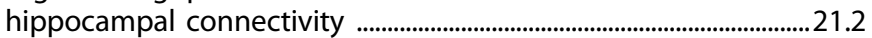

Hippocampal subfields ........................................................................... P32

Hippocampal-prefrontal ..................................................................P459

Hippocampus

11.4, 54.3, P3, P6, P7, P12,

P22, P41, P166, P264, P299,

P396, P456, P486, P528, P529,

P530, P748, P788, P807

Hispanic/Latinos

P333

Histone acetylation

P75, P461

histone deacetylase

Histone methylation

$16,16.2,16.4,16.5$

histone variants

$16,16.3$, P221, P461

historical loss

P708

HIV

.51 .2

Homer1

Homosexuality ................................................................................................. 24

hormonal contraceptive use ............................................................... P81

HPA axis .......................................................................... 10.5, P136, P783

Human Clinical trial .................................................................................... 8.3

Human Genetics

P147, P171, P746

Human Neuroimaging

41.3, P58, P79,

human postmortem brain P276, P765

human postmortem brain tissue ..............................................................29.4

Human transmission .......................................................................................30.5

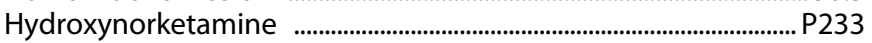

hyperalgesia ................................................................................................P676

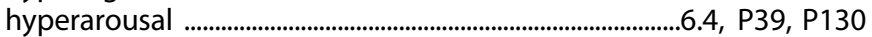

hyperthermia .................................................................................................... 4.4

Hypobaric hypoxia ............................................................................... P338

hypochondriasis .......................................................................................... 1.1

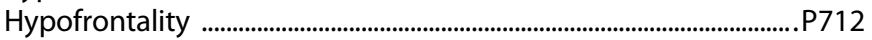

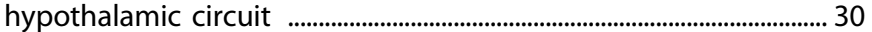

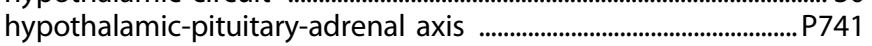

hypothalamus ………………………………...........136, P212, P559

Hypoxia ............................................................................................................22

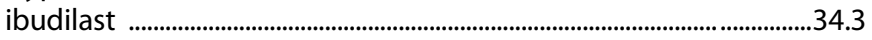

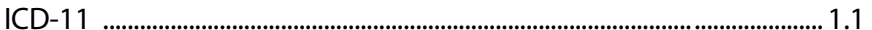

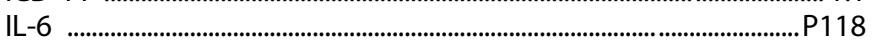


imaging

.54, P801

Imaging-genetics

... P726

immediate early gene

P612, P635

Immune

P170

Immune Biomarkers

P38, P412, P674

immune cells

P482

immune modulation

P310

Immune responses

P230, P379

immune system

P352, P830

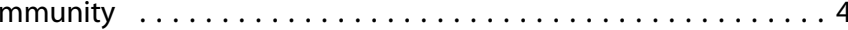

Immunity \& neurodevelopment by sex ...........................................P345

immunohistochemistry ...........................................................................P188

Implicit association test ........................................................................... P144

impulse control $\ldots \ldots \ldots \ldots \ldots \ldots \ldots \ldots \ldots$

impulsive sensation seeking .............................................................P270

impulsivity

$22,46,46.2,46.3,46.4, \mathrm{P} 90$

P153, P156, P199, P203, P208,

P304, P307, P588, P693, P761

in vitro fertilization P540

In vitro neuronal differentiation

P303

in vivo

P47

in vivo calcium imaging

11.3, P39, P40, P50,

P67, P438, P597

In vivo Imaging

$30.2,30.4,41, \mathrm{P} 422$

In vivo microscopy

50.2

incentive motivation

P697

inclusion and exclusion

P790

ndependent Component Analysis

Indirect Pathway ............................................................................... P534

individual differences .........................................................................P214

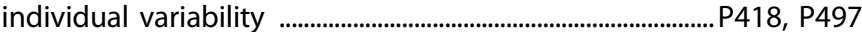

Individual vulnerability ............................................................................46.1

Induced pluripotent stem cells (iPSCs) ...............................P123, P401,

P504, P539

infant electroencephalography

P141

inflammation

44.2, 51, 51.1, 52, 52.2,

52.3, 52.4, P241, P316, P352,

P395, P412, P498, P569, P789

51.3, 51.4, 52, P545

Inflammatory Markers

... P79

Inhibition

Inhibitory control

P26, P287, P707, P801

innate behavior

P256

insomnia

20.5, 51, 51.4, P99,

P581, P606, P764

insomnia disorder ......................................................................................... P582

instrumental learning ........................................................................ P663

Insula ....................................................... 55.2, P145, P237, P456, P760

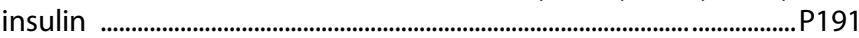

insulin resistance .................................................................. P2212

intellectual disability .............................................................................. P124

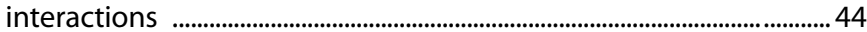

Interbrain Synochrony .............................................................................. P809

Intergenerational Transmission ....................................................... P149

Intergenerational transmission of trauma ......................................P110

Interleukin-8

P305

Intermittent Access self-administration .................... 20.3, P643, P659

internalizing disorders ............................................................ 54.4, P822

Interpersonal Violence ........................................................................ P63

inter-subject correlation .................................................... P625

intravenous drug self-administration ............................... P624, P642

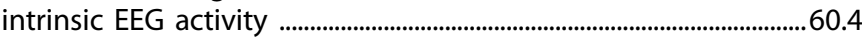

intrinsic excitability .................................................................................29.2

intrinsic motivation ..............................................................................P571

ion channels .................................................................. P431, P539

irradiance ...............................................................................................P28

irritability .......................................................47, 53, 53.1, 53.2, 53.3, P674

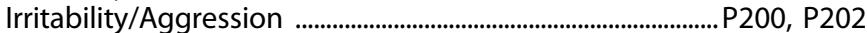

IV- Ketamine ..............................................................................................P2 juvenile critical period

P108

juvenile social isolation

P787

Kaplan-Meier Survival Curve

P1

kappa opioid receptor

45.2, P278, P379, P766, P779

Kappa Opioid Receptor Antagonist

Ketamine

P731

25.2, 50.3, P66, P68,

$\mathrm{P} 102, \mathrm{P} 233, \mathrm{P} 293, \mathrm{P} 328$,

$\mathrm{P} 400, \mathrm{P} 402, \mathrm{P} 405, \mathrm{P} 458$

Ketorolac P102

kinase inhibitor

P344

kinome array

P550, P559

Kinomics

P322

kinship

$11,11.2$

kratom

P667

Kv3 channels

Kynurenic acid

P460

Kynurenine pathway

L1000

$6.3,6.4,6.5$, P557, P572

$6,6.3,6.4,51.3$

Lactate

Language

Late-life Depression

P262

P284

P496, P654

8.1, 8.2, P9, P11,

P227, P248, P354

latency of acoustic startle

P545

Latent class analysis

Lateral Habenula

Lateral hypothalamus

$P 238, P 289$

lateral septum

Latin America

194, P293, P752

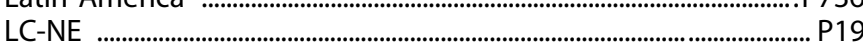

Learning Health System ..................................................................... P555

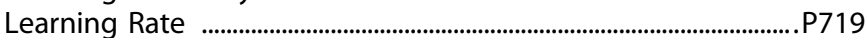

lemborexant ....................................................................................................P585

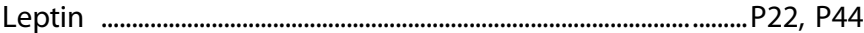

Leptin Resistance ............................................................................................. P509

leukocyte telomere length ...................................................................... 4.3

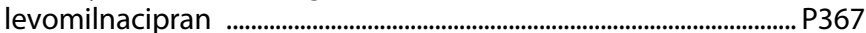

LgDel ....................................................................................................... P570

Lifetime stress ............................................................................ 44.3, P456

linguistic markers ................................................................................ P98

lipid oxidation .......................................................................................... P567

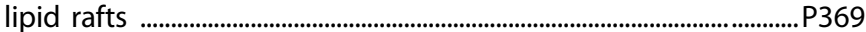

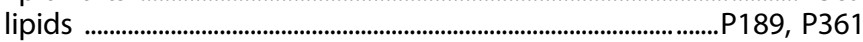

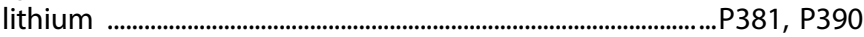

local field potentials .........................................................................................5.

Locus coeruleus (LC) ............................................................................. P9 P10

Long Access self-administration .............................................................20.2

Long Term Potentiation .......................................................................... P462

Long-acting injectable antipsychotics ............................19, 60.3, P541

longitudinal analysis ................................................................ 26.2, P135

Longitudinal MRI .................................................................. P107, P810

longitudinal study ...................................................................... P85, P763

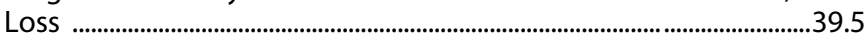

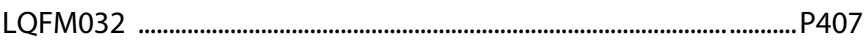

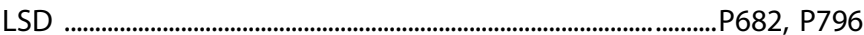

LSD microdosing .......................................................................... P818, P824

lumateperone ...................................................................................P319

M5 muscarinic receptor ......................................................................... P330

Macaque

$\mathrm{P} 165, \mathrm{P} 794$

machine learning

21.4, 39.4, P112,

P318, P474, P496

machine learning classification ......................P198, P421, P473, P542

Machine learning clustering .........................................................................4.4.4

Macrophage .............................................................................................52.2

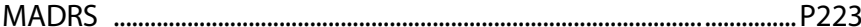

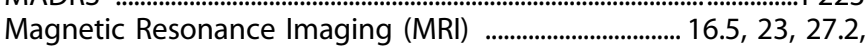

37.3, P34, P115,

P156, P261, P364, P392

magnetic resonance spectroscopy ....................................... 34.3, P163, 
Magnetic seizure therapy

P176, P404, P520

P289

magnetoencephalography

Major depression

Major Depression Disorder

P454, P531

P215, P226, P396, P575

...P281, P314, P322,

P350, P384, P389, P401

Major Depressive Disorder (MDD) ................................21.2, 21.3, 31.5, P51, P66, P83, P94, P179, P218, P222, P223, P224, P235, P241, P245, P246, P251, P259, P264, P271, P301, P303, P305, P312, P326, P353,

P357, P359, P371, P374, P415, P819, P392

maladaptive feeding . P190

mania P270, P341, P505

marijuana P616, P653

Marijuana Policy P481

marmoset P13

mast cells

... 4.1

MAT

P701

maternal brain

P690

Maternal Depression

. .26 .4$

maternal immune activation

P463

maternal sensitivity

P690

maternal separation

P134

maternal stress

P151

Matrix Metalloproteinase-9 (MMP-9)

P628

MDMA

2.3, P682

mechanism-based biomarkers

$4,4.2,5$

mechanisms

.... 37

Medial Orbitofrontal Cortex

35.4, P332, P785

Medial Prefrontal Cortex

$22.4,45,45.2$,

50.3, P12, P89, P109, P195,

P213, P300, P537, P599, P612,

P659, P795, P802, P808, P830

Medial septum P710

Medical Marijuana

P606, P633

medication development $\ldots \ldots \ldots \ldots \ldots \ldots \ldots \ldots \ldots \ldots$

medication-naive first episode psychosis ...........................................60.2

medications 50.2, P67, P190, P655

Medium Spiny Neuron

MEF2C

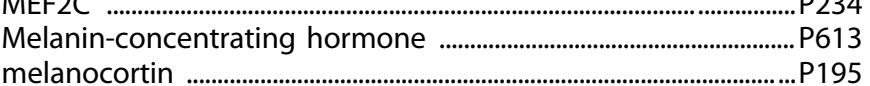

memantine

membership

P790

Memory

4.3, P3, P49, P71, P406, P748

Memory and Learning ... P15

Memory Encoding and Retrieval .......................................................P663

Memory engram cell

P75, P528

memory reconsolidation

menopause P768

mental disorder

$4.3,56.3$, P18

Mental health disorders ...................................................................... P181

mental health service use ...................................................................... P827

mental illness ..................................................................................................... 3.1

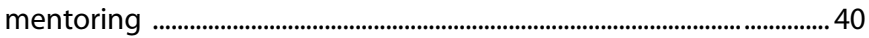

mesolimbic reward circuitry

P182, P190, P592,

P617, P730

meta-analysis P97, P133, P520, P742, P812

metabolic function ... P53

metabolism

.P648

metabolites

metabolomics

Methamphetamine

P189, P349

P624, P652, P705, P712

methamphetamine seeking .................................................................P641

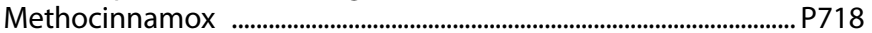

methodologies $\ldots \ldots \ldots \ldots \ldots \ldots \ldots \ldots \ldots \ldots \ldots \ldots \ldots$ methylphenidate

mgluR5

P55, P505, P654, P679, P699

mice

P51

microbiome

$\mathrm{P} 166, \mathrm{P} 815$

microbiota

$28.2,33,53$, P334

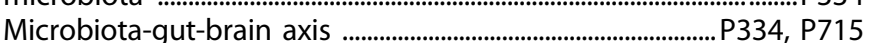

Microdialysis

microglia

...P650

28.2, 44.4, 52.2, P24,

$\mathrm{P} 114, \mathrm{P} 129, \mathrm{P} 278, \mathrm{P} 306, \mathrm{P} 360$,

P379, P416, P558, P624, P644

microglia engulfment 54.1

microPET ................................................................................................ P134

MicroRNA ……….............................................................................................P747

midbrain ...............................................................................................P122

mild cognitive impairment due to AD .............................................P314

Mild Traumatic Brain Injury ........................................................................... P88

Mindfulness Meditation ..........................................................................P145

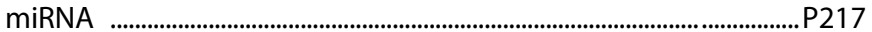

Mirror Neuron .............................................................................................P528

Mismatch Negativity ...............................................................P464, P514

Mitochondria …..............................P23, P244, P255, P275, P416, P637

Mitochondrial DNA ..................................................................................

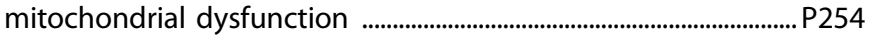

mitochondrial genetics ......................................................................... 44

Mitochondrial Respiration ..................................................... P23, P302

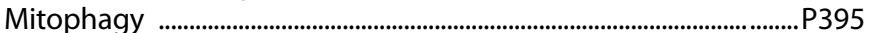

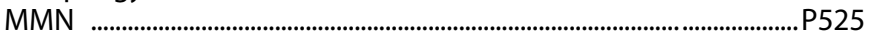

model systems .............................................................................................. P706

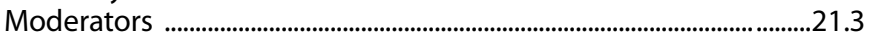

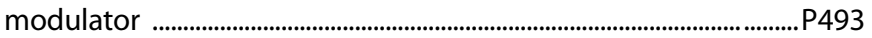

Molecular imaging ..................................................................................16.5

molecular neuroscience ..................................................................173, P293

Molecular profiling ......................................................................................... 11.1

monetary incentive delay task ...........................................................P193

monkey

P380

monoamine oxidase B

$\mathrm{P} 425, \mathrm{P} 767$

Monoamine Transporters ..............................................................................221.1

Monoiodoacetate Model of Osteoarthritis .....................................P432

Mood

P285, P391, P415, P738

Mood and cognition

P668

mood disorders

P121, P217, P237,

P298, P345, P364, P373, P385

morphine

$P 592, P 745$

Mortality

...11, 11.4

motivated behavior

4, P534

motivation

...P780

motor activity ...................................................................................................... 121

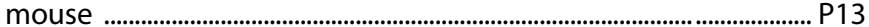

mouse genetics ...................................................................................... P735

Mouse models ................................... 28, 34.4, P292, P295, P426, P596

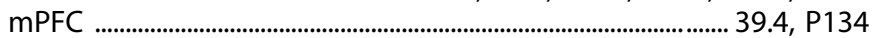

MR spectroscopy ...........................................................P385, P483, P538

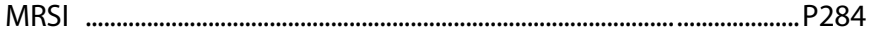

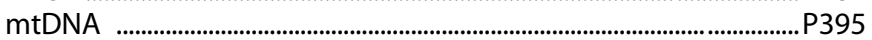

$\begin{array}{ll}\text { mTORC1 } & \text { P273 }\end{array}$

Multi-electrode arrays ........................................................................................... P47

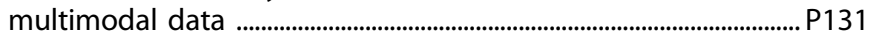

Multimodal Neuroimaging ..........................................................................37.5

Multi-omics ..........................................................................................P349

multivariate .............................................................................................P106

multivariate analysis ..................................................... 38.3, P469, P703

mu-opioid receptor agonist ................................................................P667

mu-opioid receptor binding potential ...............................................P391

mu-opioid receptors

42.2, P339, P600,

P732, P819

Muscarinic agonist

P510

muscarinic

M4 P493, P500 
MVPA

Myelin Imaging

P107

Myelination

14.1

$\mathrm{N}$-acetylcysteine

P628

$\mathrm{NACHO}$

P617

naloxon

P750

Narcissistic traits

P202

native americans

$51.2, P 287$

Natural Compound

P365

natural language processing (NLP)

P2, P465, P496

naturalistic drug cues ................................................................................ P625

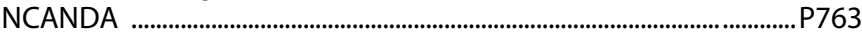

$\mathrm{N}$-desmethylclozapine .................................................................... P568

near-infrared light

P280

Negative affect

46.3, P730

negative allosteric modulator

P623

negative emotionality

P605

negative reinforcement

P408

negative symptoms

Negative urgency

P480, P498, P546, P554, P821

negative valence

46.3, P304

Negative Valence System ............................................................... 37.4. P385

Neighborhood Crime ........................................................................... P168

Neighborhood Poverty ................................................................................ P65

Network activity ........................................................................... 30.2, P331

Network Modeling ................................................................................ 3.2

network neuroscience ................................................................... 3.2, P791

Network-Analysis ............................................................................................ 3.2

Neural circuit and animal behavior ....................................................P683

neural circuitry ............................................................................... 128, P820

neural circuits ……………………...........................30.3, P308, P346

Neural connectivity .................................................................... 14.2, P184

Neural coordination ..............................................................................P662

Neural network connectivity .............................................................P140

Neural networks ........................................................................ P63, P799

neural oscillations ................................................................. 57.3, P198

neural plasticity ..................................................................................... P459

Neural Predictors ....................................................................................... P494

neural progenitor cells .........................................................................P303

neurite density ................................................................................................. 14.1

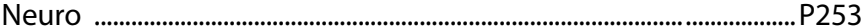

neuroactive steroid ...................... P222, P223, P224, P235, P271, P283

neuroanatomy ................................................................P201, P441, P570

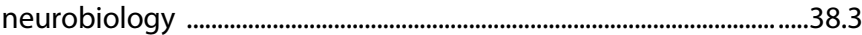

neurocircuitry ............................................................................................... 47

neurocognition ...........................................................132, P341, P469

Neurocognitive functioning ....................................................................P687

neurodegeneration ............................................................................... P427

Neurodegenerative Disease .......................................................56.4, P5

neurodevelopment ....................................28.4, 38.2, 44.5, P107, P116,

$\mathrm{P} 151, \mathrm{P} 186, \mathrm{P} 486$

neurodevelopment $\&$ aging $\ldots \ldots \ldots \ldots \ldots \ldots \ldots \ldots$

Neurodevelopmental Disorders ....................... 16, P105, P120, P124

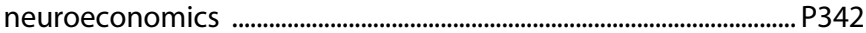

Neuroendocrine .......................................................................................... 10.2

Neuroendocrine responses ................................................................... P783

neuroendocrinology ..............................................................................53.3

Neurofeedback ...............................................................43, 43.2, P447, P473

Neurofibrillary tangle .........................................................................P782

Neurogenesis ...................................................................... 11.1, 52.2, P529

Neurogenesis enhancers ..................................................................... P56

neurogenetics ..........................................................................................38.2

Neuroimage

...P631

neuroimaging

$2,7,23.4$

neuroimaging biomarkers

neuroimmune

21.2, P386, P506, P803

31, P329, P734

neuroimmune activation

6.2, P592, P611, P781

neuroimmune interaction neuroimmune mechanisms

$31.3, \mathrm{P} 178$

neuroimmune mechanisms: prenatal to aging $\ldots \ldots \ldots \ldots 4$

Neuroimmunology

P416

Neuroinfection

P411

neuroinflammation

$4.1,6,23,34,52.5$

$\mathrm{P} 29, \mathrm{P} 161, \mathrm{P} 180, \mathrm{P} 305, \mathrm{P} 422$,

P530, P543, P624, P770, P815

Neurokinin

$P 263$

neuromarkers

31.5, P749

Neuromelanin-sensitive MRI P9, ……........................................ P10

Neurometabolism ....................................................................................... P820

Neurometrics ........................................................................................P511

neuromodulation .........................................................1, 30.5, P280, P759

neuromodulators ......................................................................................... 42

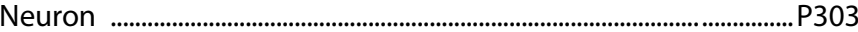

neuronal ensemble ...................................................................................4 45.4

Neuronal Epigenome ................................................................... 41.2, P108

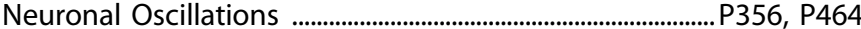

Neuronal stem cells (NSCs) ................................................................P401

neuron-derived exosomes (NDE) ........................................................P248

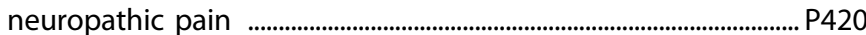

neuropathology

$23.2,48.3,48.4$

neuropeptides

39.2, P212, P263, P764

neuropharmacology

$P 209, P 308$

neurophysiology

P177, P215, P384, P724, P792

Neuroplasticity

2.3, P320, P525, P811

neuroprotection

P424

Neuropsychiatric Disorders

Neuropsychiatric Disorders [Schizophrenia,

Parkinson's Disease, Major

Depressive Disorder]

$56.1,57.4$, P428

Neuropsychiatric sym

57.5, P461, P530

neuropsychiatry

48.4, P791

neuropsychopharmacology

P328

Neurosteroid

Neurostimulation

P784

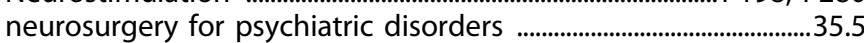

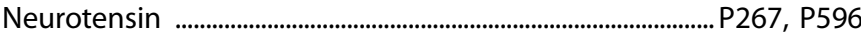

neurotransmitter co-release ...............................................................P380

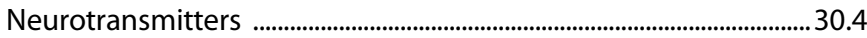

Neurovascular coupling .......................................................................... P148

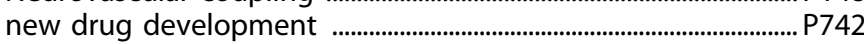

next generation sequencing ...............................................................P411

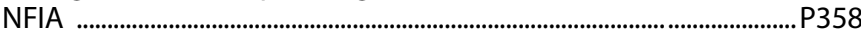

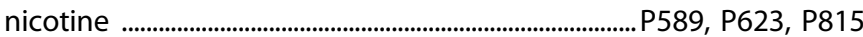

nicotine addiction ....................... P617, P623, P660, P670, P716, P781

nicotine dependence .................................................................P475, P706

nicotine vapor ............................................................................................. P706

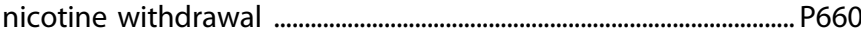

nicotine/substance use disorder .......................................................... P642

nicotinic acetylcholine receptors ................................. 617, P642, P658

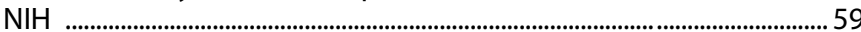

NIH HEAL Initiative ............................................................................... P432

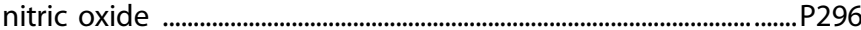

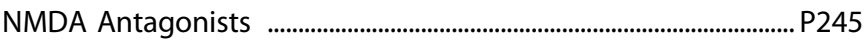

NMDA glutamate receptors ...................................................P246, P311

NMDA Receptor ............................................6.5. P431, P471, P525, P574

NMDAR antagonist .............................................................................. P239

NODDI .

non pharmacological interventions ......................................................55.4

noncoding RNA ........................................................................ P634, P744

Nonhuman Primates .......................................................35.2, P78, P201

non-invasive brain stimulation .............................................. 55.3, P232

Non-invasive Neuromodulation ............................................P384, P729

Non-opioid, Non-addictive Therapeutics ..............................P429, P432

non-parametric item response theory ...............................................P516

Non-Suicidal Self-injury (NSSI) P115

noradrenergic .....................................................................................................P126 
Noradrenergic System

56, P681

norepinephrine P10, P437, P691

novel antidepressant ... P56

novel assessment tools for clinical trials

Novel coronavirus (SARS-CoV-2)

...P160

novel object recognition

Novel Targets

novel therapeutics

novelty response

novelty seeking

Nrxn1-alpha

nuclear factor kappa B

Nucleus Accumbens

23.2, P411, P419, P424

56.4

.P563

15, P83, P283, P618, P753

. P641

P64

4.1

P48

29.2, 29.3, 29.4, 34.2, P128,

P190, P207, P221, P230, P294, P342,

P352, P387, P598, P621, P626, P629,

P637, P655, P665, P743, P781, P823

nucleus accumbens core ..P649, P713

nucleus reuniens

P268

Nucleus tractus solitarii P698

Obesity

P191, P194, P197, P240

Obesity and eating disorders

.46 .3

Obsessive Compulsive Disorder (OCD) ........................ 1.3, 35.4, 35.5,

$50,50.2,50.4,50.5, \mathrm{P} 433$,

$\mathrm{P} 436, \mathrm{P} 437, \mathrm{P} 438, \mathrm{P} 440, \mathrm{P} 442, \mathrm{P} 443, \mathrm{P} 444$

Obsessive-Compulsive and Related Disorders ................................... 1.2

Obsessive-compulsive personality traits ..............................................P434

obsessive-compulsive spectrum disorders (OCDS) ............................ 1.3

Obsessive-compulsive symptoms ............................................................ 1.1

obstructive sleep apnea ..............................................................................P585

OCD phenotypes .................................................................................... P434

older adults ........................................................ 8.4, P29, P231, P406

ondansetron ......................................................................................................... 17.2

ontogeny .......................................................................................................... 11

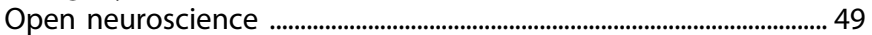

Operant behavior ...............................................................P608, P725, P743

opiate addiction ...................................................................................P765

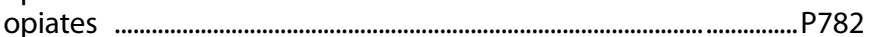

Opioid abuse ..........................................................P601, P608, P612, P627,

$\mathrm{P} 650, \mathrm{P} 688, \mathrm{P} 732$

Opioid addiction ............................................... 20, 20.3, 20.5, 42.3, 45.5, P429, P588, P592, P594, P601,

P602, P665, P715, P738, P744, P746, P779

Opioid antagonist treatment ................................................................ P16

opioid dependence ........................................................................... P730

Opioid peptides ........................................................................... 45, P779

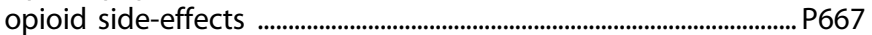

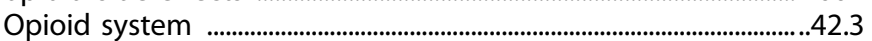

opioid use disorder .........................................................20.2, 20.4, P583,

P621, P690, P694, P731, P735,

$\mathrm{P} 748, \mathrm{P} 753, \mathrm{P} 765, \mathrm{P} 819$

opioid withdrawal ............................................................ 20.5, P615, P676

opioids

42, P16, P701, P727,

P728, P765, P776

OPRM1

Optimism .................................................................................................

optogenetics .............................................................. 11.3, 39, P39, P216,

P337, P743, P768

oral antipsychotics

orbitofrontal cortex (OFC)

22,3, 50.4,

P622, P645, P663, P684,

P702, P785, P821

orexin

20.4, P659

orexin receptor

P659

orexin receptor antagonist

20, 20.4, P188

orexin/hypocretin

$20.2,20.3$

organic cation transporters

P671 outbred rats

P677, P739

ovarian hormones

53.1, P351

oxidative DNA damage

P381

oxidative stress

P211

oxycodone

20, P694, P721

oxytocin receptor P157

p factor

P135

PACAP

P35, P553, P649

Pain

30, 30.3, 42, P176, P408,

Pain sensitivity

P606, P612, P633, P701

Pain therapeutics

$P 161, P 366, P 412$

pair bond

P420, P805

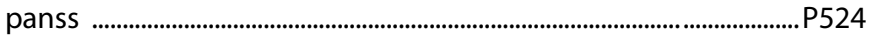

paradigm ................................................................................................38.

parahippocampal place area (PPA) _.................................................... P551

paraventricuar nucleus ..........................................................................P136

paraventricular nucleus of the hypothalamus ...............................P683

paraventricular nucleus of the thalamus

$P 267, P 621$

Parent - child dyads

26, 26.2, 26.3, P206

parietal cortex

P816

Parkinson's disease

P265, P426

Parkinson's Disease and Dementia Psychosis .................................P426

partial agonist ............................................................................................ P667

Partial Agonist Ligands .........................................................................P590

Parvalbumin fast-spiking GABAergic interneurons .........P599, P609

parvalbumin interneurons.

P712, P808

Parvalbumin neurons

P300, P460, P484, P673

PASC

P240, P494, P555

Patient outcomes

……................ P56

Pavlovian conditioning ................................................... P27, P626, P705

Pediatric Bipolar Disorder .....................................................................P393

pediatric irritability

P37, P403, P797, P799

Perceived stress

P211, P586, P678, P700

Perceptual decision-making

P5 535

periaqueductal grey (PAG)

P607

Perimenopause

$10.5,53.3$

Perinatal stress 44.2

Perineuronal nets P609, P673, P712

Peripheral Biomarker ................................................................... P355

Peripheral blood marker ....................................................................... P542

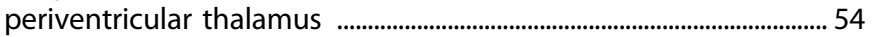

personal space .......................................................................................... P816

personality ...................................................................................................P682

personalized medicine ...................................................21, 21.1, P595

Pesticides .................................................................................................44.2

PET Imaging ........................................P10, P19, P210, P391, P668, P716

PET Imaging Study ................................................................................... P51

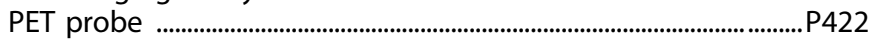

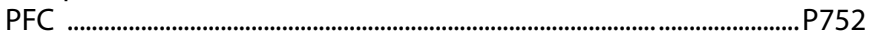

pharmacoBOLD …………………………………………...... P209, P458

Pharmacodynamics .............................................................................. P514

Pharmacoepidemiology ..........................................................................P120

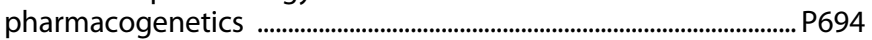

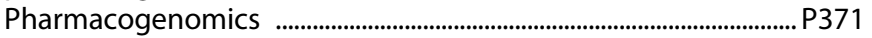

Pharmacokinetic and Pharmacodynamic ......................................P273

Pharmacokinetics .................................... P159, P518, P716, P742, P805

Pharmacology .......................................................................... 17, P209

pharmacotherapy .............................. 1.3, 17.1, P443, P630, P639, P730

Phase II clinical trial ............................................................................P429

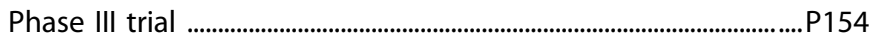

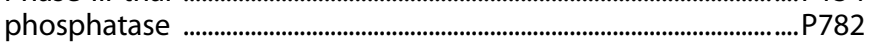

Phosphodiesterase-4 (PDE4) …………………….......34, 34.2, 34.4, 34.5

photobiomodulation .....................................................................................P28

pimavanserin ............................................................................................. 5518 
PKCdelta

P86, P711

placebo-controlled trial

$32, \mathrm{P} 824$

placenta

28.4, 44.2, P118

Polydrug Use ..P587

Polygenetic Risk Score

P242, P383, P450, P477, P695

polygenic risk score

polygenic risk scores

38.3, P132, P363, P540, P819

Polygenic scores

$25,28.5,52.3$, P299, P575

Polypharmacy

13.5

polysomnography

..P390

polysubstance abuse

P402, P557

Poor clinical outcome ...................................................................... P275

Population genetics ....................................................................................38.4

Positive Affect ...............................................................................................26.3

Positive symptoms .................................................................................... P554

positive valence

P442

positron emission tomography (PET) ..................... 7, 13.4, 16.4, 41.1, P165, P291, P425, P500, P526,

$\mathrm{P} 729, \mathrm{P} 766, \mathrm{P} 767, \mathrm{P} 819$

Post Traumatic Stress Disorder (PTSD) ........................ 2.3, 17.1, 31.4 31.5, 39.2, 41, 41.1, 41.2, 43, 43.2, P31, P38, P48, P49, P51, P53, P57, P59, P61, P65, P66, P67, P70, P79, P83, P91, P93, P94, P97,

P98, P99, P100, P167, P247, P382

Posterior Cingulate Cortex P145

Postmortem P532, P549, P567

post-mortem brain ... 521

Postmortem Brain Tissue

P94, P264, P468

postoperative cognitive dysfunction ................................................. P16

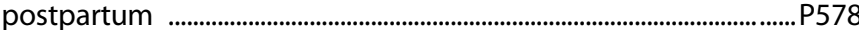

postpartum depression .......................................................................... P351

postpartum mood ..................................................................................... P141

posttraumatic stress ………………...................................................... P29

potential mechanism ................................................................................... 13

pragmatic analysis .......................................................................................... 8.2

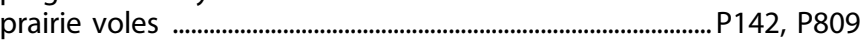

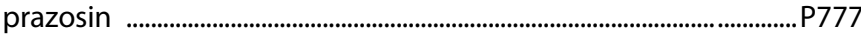

Precision Medicine for Depression .....................................................P810

Precision Medicine for Mood and Anxiety Disorders ................P159

Precision Medicine for Mood Disorders ...............................P310, P355

Preclinical pharmacology ...................................................................... P563

Preclinical Screening Platform for Pain ...............................P420, P432

precuneus ......................................................................................... 284

Predictive Biomarker .................................................................................. P70

predictive coding ........................................................................................... 2.4

Predictive Models ...................................................................................... P250

prefrontal circuit maturation ............................................................... P13

prefrontal cortex ..........................................35.3, 45.5, 57.2, P78, P295

P342, P344, P448, P464,

P543, P594, P635

Pregabalin P727

pregnancy

23.4, 53.1, P151, P168, P178

prelimbic cortex P33, P36, P40, P578

P649, P666, P713, P724, P745

premenstrual dysphoric disorder ......................................................P335

premenstrual syndrome ............................................................................. P335

Prenatal alcohol exposure ........................................................................ P138

Prenatal Drug Exposure .....................................................................P164

Prenatal exposure ..................................................................... 4.3, 28, P142

Prenatal immune exposures ................................................P106, P543

prenatal stress ............................................28.2, 28.3, P118, P139, P234

Prepulse Inhibition

. P25

pre-school children

Prescription Opioids

9.2

prevention

P182, P408, P746

probabilistic reversal learning
Processing speed

P487

prodromal schizophrenia

P545

prodrome

Prodynorphin

11.4, P731

progesterone

P351, P820

Progressive ratio testing

P643

Pro-inflammatory cytokines

projection targets

31.3, 34.3, 52.2, P411

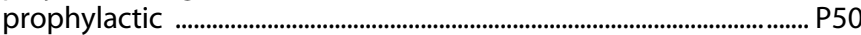

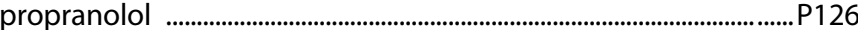

prostaglandin .................................................................................................P211

protein aggregation ............................................................................... P449

protein kinase activity .....................................................................P550

Proteomics

41.1, P93, P318, P322, P355,

P549, P591, P688, P765

Proteostasis

P449

proton magnetic resonance spectroscopy

P307

provider perspectives

8.4

Psilocybin

22.4, P181, P229, P350,

P353, P654, P784, P796

Psychedelic medicine

2.5, P229

Psychedelics

2, 2.4, 15, P181, P225,

P348, P365, P382, P627,

P654, P796, P800, P818

Psychiatric Comorbidity

37.4, P177, P307, P703

psychiatric genetics

P428

psychiatric measurement ..................................................................... P100

psychological distress

... P81

Psychological Wellbeing

8.3, P231

Psychopathology

37, P542, P798

psychophysiology

psychosis

$6.2,13,13.2,13.4,38,38.2$

52, 52.3, 60.4, P6, P473, P476,

P478, P481, P482, P483, P495,

P520, P532, P564, P572, P573

psychosis risk P571

psychosocial intervention

psychosocial stress

P156

Psychostimulant

P325, P329, P607

Psychotherapy

P505, P677, P782

Psychotic Disorders

2, P448

Psychotic-like experiences ............................................................ P511

PTSD depression ...................................................................... 15, P18, P72

Pubertal stress

$\mathrm{P} 113$

Puberty

P147, P186

punishment

22.3, P640, P643

pupillometry ............................................................................................P792

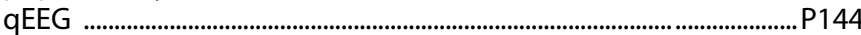

QTL

qualitative research ...................................................................... 8.4, P333

Quality of Life .........................................................................................P633

Quantitative Electroencephalography (qEEG) ..........................26, P384

R-(-)-ketamine ......................................................................................P405

Race disparities ............................................................................. 23.5, 27.2

racial differences .............................................................................. 27, P741

racial ethnic minority .............................................................................. 12

Racism

$27.1,27.3,59$

Racism and discrimination stress

28.3, P169

Randomized-Controlled Trial

55.5, P350, P502

raphe nucleus

P426

Rapid Antidepressant

P235, P283

Rapid Antidepressant Effects ................................................. P246

Rapid Cycling ...........................................................................................P375

Rapid depression treatment .................................................................... 15

Rare genetic variants ..................................................................... 38.2, 48.1

Rare genetic variation ......................................................................... P445

Rare Neurodevelopmental Disorders ....................................P105, P123 
Rat models P584

Rating scales

P516

rats

P192

RCT 2,

17.4

real-time $\mathrm{fMRI}$ neurofeedback

43, 43.2,

43.3, 43.4, P122, P145, P467

recognition memory .................................................................. P166, P641

recordings

Reinforcement learning

P745

reinstatement

P403, P721, P815

REL-1017

P717

relapse

P315

relapse and treatment outcome

13.3, 19, P602, P613, P673

relapse biomarkers

P605

Relevance for Outcomes

60.3

REM sleep

... P555

remifentanil

6.4, P35, P613

remote assessment solution

P608

Repeated treatment

P228

repetitive transcranial magnetic stimulation (rTMS) ........55.4, 55.5,

P215, P232, P384,

P418, P497, P502, P533

reproducibility ... P106

reproduction

$10.3, \mathrm{P} 410$

Research domain criteria (RDoC) 37, 37.2, P200, P595,

P783, P786, P817, P828

resilience

41, 41.2, 41.3, P98, P715

Respiration

P727

respiratory safety

P585

response inhibition

P702, P797

Resting and task fMRI

... P88

resting state brain dynamics

...P389

Resting State Connectivity

P104, P631

Resting State Functional Connectivity

21.2, 35.2,

60.2, P64, P76, P110, P112,

P137, P169, P214, P251, P285,

P323, P358, P475, P503,

P511, P527, P699, P796

P261, P282

Resting state intrinsic connectivity

P74, P218, P506, P784

Resting-state fMRI

P715

Resveratro

P511

Reliability

35.2

retrograde tracing

reversal learning

Reward

1.4, 14, 14.4, 22.5, 29.4, 42.4,

P213, P308, P357, P387,

P699, P704, P772, P780

reward and aversion ............................................................................ P651

Reward Anticipation ............................................................................ P270

reward circuitry ........................................................................................................ 29

reward deficit ............................................................................................P128

reward devaluation ...................................................................................... P724

Reward functioning .................................................................P241, P783

reward neural circuitry ........................................................................P216

reward prediction error ........................................................................... P403

Reward processing ........................................................P393, P561, P648

Reward Sensitivity ................................................................................... P771

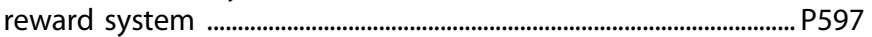

Reward-based decision-making ..............................................................25.2

reward-seeking behavior ...................................................................... P745

Rhesus monkeys ..........................................................P645, P691, P718

RiboTag ................................................................................................P644

right temporo-parietal junction ........................................................ P527

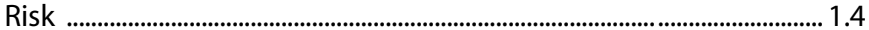

Risk and Resilience ...................................................................... P113, P119

Risk Calculator ............................................................................................... P363
Risk Evaluation and Mitigation Strategy (REMS)

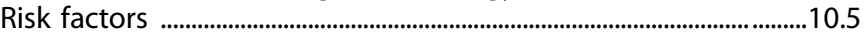

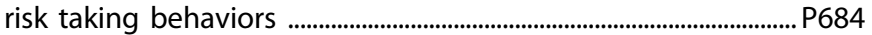

risk-taking ...............................................................................................P695

Risky decision-making ..................................................................... P640

RNA binding protein ...................................................................................... P15

RNA Sequencing ........................................................................P300, P723

RNAscope fluorescence in situ hybridization ...................... P45, P484

RNAseq ........................................................... P78, P236, P276, P351,

P598, P644, P772, P794

rodent

P617

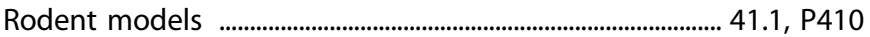

rsfMRI functional connectivity ............................................................P368

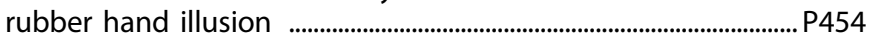

rumination ................................................................................... 21.3, P389

Safety ..........................................................................................................P224

Salience ....................................................................................................P112

salience network

$55.4, \mathrm{P} 201$

SAPAP3 50, 50.3

SAPAP3 KO mice ................................................................................ P442

scanner harmonization .........................................................................P487

Schizophrenia (SCZ) ................................................. 6, 6.4, 13.5, 19, 28.4,

$32,33,38.4,38.5,48,48.1,48.2$,

$52,52.4,60.2, \mathrm{P} 423, \mathrm{P} 449, \mathrm{P} 451$,

P456, P457, P458, P460, P463, P465,

P467, P470, P475, P478, P479, P484,

P486, P487, P491, P493, P494, P495,

P496, P497, P498, P499, P500, P502,

P504, P510, P512, P514, P517,

P518, P521, P532, P533, P536, P537,

P539, P541, P543, P546, P549, P550,

P551, P552, P554, P561, P563, P566,

P567, P568, P569, P575, P576, P577, P755

schizophrenia and bipolar disorders

P477, P544, P803

schizophrenia negative symptoms

$P 491, P 560$

Schizophrenia novel treatment

55.5, P472, P507,

P553, P579

schizophrenia spectrum disorders

P453, P469, P509

Schizophrenia subtypes

.52 .5

Schizophrenia-like Behavior

P405, P471

selective attention

P551

Selective Serotonin Reuptake Inhibitors (SSRIs) .................. 2.5, P159,

P259, P262, P367

Self-Administration

25, 34.5, 45.4, P584,

P596, P623, P647, P656,

P701, P705, P718, P721

Self-esteem

..P391

self-injury

...P817

Self-medication with psychedelics ................................................P181

self-other processing ...43.4

self-referential

P817

self-referential processing

2.4, P243

Sensitive Period

28, 54.2, P446

Sensitivity

P686

Sensory Processing

Septal area P419

sequencing

11.1

serious mental illness

Serotonin

48.1, P566, P575

P773

$30.4,56,56.2, P 45, P 47$,

P192, P229, P345, P645, P691, P751

Serotonin 5-HT2A Recepto

P192, P259, P317, P645

Serotonin 5-HT2C Receptor ...................................................... 42.1, P192

serotonin and norepinephrine reuptake inhibitor ..........P262, P367

Serotonin Transporter .......................................................................P2 10

Sertraline .....................................................................................................P371 
severe mental illness

sex

sex differences

$4,4.3,10.2,22.2,25.1$ 41, 58, P7, P8, P23, P70, P73, P80, P81, P148, P150, P220, P269, P285, P294, P301, P317, P340, P360, P370, P466, P583, P615, P621, P640, P643, P651, P658, P684, P685, P692, P710, P766, P781, P809

Sex Hormones P80, P416, P762

sex pairing ...P757

sex-specific

P417

Sexual Dimorphism

$4.2, \mathrm{P} 2$

Shank3-deficient mic 16.2

Sigma2, $5 \mathrm{HT}$, Alpha receptors

P546

sign-tracking

P626, P705

Simultaneous PET-MR

P388, P699, P709

Single cell

... P91

single cell omics

P94, P611

Single-cell RNA sequencing

11.1, 29, 42.2,

P95, P138, P290,

P339, P688, P689

single-cell sequencing .29 .4

single-nucleus RNA-seq P264, P276

Skin conductance responses ... P31

SLC39A8

P451

SLC6A1/GAT1

P423

sleep

sleep architecture

P11, P285, P402, P409, P586

Sleep disturbances

P581, P582

Sleep inconsistency

P72, P583

sleep spindles P632

slow oscillation

P472, P522, P557

wave sleep

smartphone ...........................................................................................P214

Smell P680

smoking

$17.4,55.2$

Smoking Cessation

$17.3,55, \mathrm{P} 668, \mathrm{P} 670$

SNP

SNP variation .................................................................................................P445

sociability .....................................................................................................P808

social anhedonia ............................................................................... P515

social anxiety

Social Behavior

$11.2,11.3,26, \mathrm{P} 33, \mathrm{P} 129$

P212, P309, P331, P395,

P661, P662, P795, P830

social cognition

P469, P578, P807

social defeat

P607

Social defeat stress

P249, P352

Social Deficits

$16.2, \mathrm{P} 154$

social determinants of health

$12,23.5$

social dominance

P309

Social Factors and Functioning

. P57

Social hierarchy

Social Interactions

39.4

social isolation

14.3, P126, P757, P802

39, 39.3, P672

social isolation stress

39.2, P594, P787

social motivation

$39,39.3$

Social rejection

P71, P325, P391

social status

............P317

social stress

P252, P309

Social support

P484, P666

Somatostatin

P529

spatial memory

P96, P264, P611

.

speech development

P141 splice variants

P396

Startle

P242, P786

statistical methods

$P 250$

stem cells

P303

Stimulants

P198, P728, P776

stimulus enhancement ............................................................................P750

stimulus inhibition ................................................................................... P750

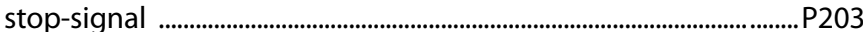

Stress ....................................P68, P168, P213, P336, P370, P388, P396

Stress abnormalities .................... P268

Stress and anxiety behavior ......................................................P344, P683

Stress and Depression .........................................................................P263

Stress and Trauma ...................................... 28.3, P57, P62, P153, P696

Stress Coping ...............................................................................P632, P683

stress hormones .................................................................................. P622

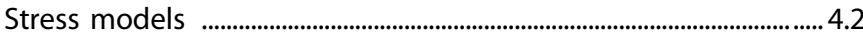

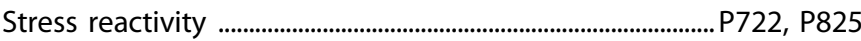

stress resilience .....................10.3, P68, P266

P295, P320, P346

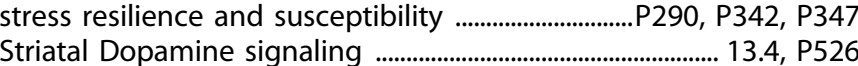

striatal functional connectivity ...............................................................60

striatum

50.2, P27, P191

$\mathrm{P} 282, \mathrm{P} 330, \mathrm{P} 532, \mathrm{P} 617$

Structural Connectivity

P197

Structural MRI 44.5, P42, P70, P162, P474

structural racism .... 12

Subcortical Shape Analysis ..............................................................P486

Subcutaneous Fat ................................................................................. P453

subgenual anterior cingulate ............................................................ P430

Subjective Response ............................................................. P685, P734

subliminal emotion ....................................................................................... P115

Substance abuse ......................................................................P556, P760

Substance abuse disorders ......................................... 10.3, P652, P719

Substance P ............................................................................................... P52

substance use disorder

23.3, 29, P610, P627,

P669, P696, P714,

P728, P737, P739, P773

Substance-related disorders …............P677

Subtypes of substance use disorder ................................................P595

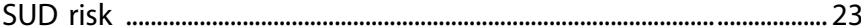

SUD-like phenotypes ................................................................................... P761

Suicidal behavior ............................................................................ 21.3, P296

suicidal ideation ..............................................................P237, P247, P364

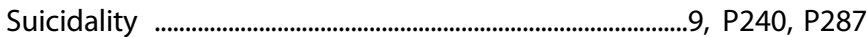

suicide ............................................ 9.3, P34, P133, P144, P313, P402

Suicide attempt ...................................... P184, P349, P358, P364, P376

Suicide prediction .....................................................................P131, P364

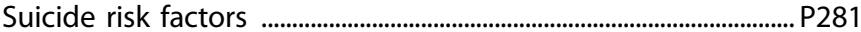

Superresolution and deconvolution microscopy .............................30.5

supplementary motor cortex ............................................................... P533

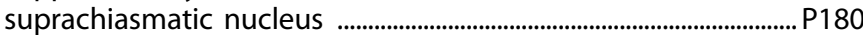

sustained threat of shock ..................................................................... P185

switching strategies $\ldots \ldots \ldots \ldots \ldots \ldots \ldots \ldots \ldots$

symptomatology ......................................................................................... P564

synapses

$\mathrm{P} 23, \mathrm{P} 265$

Synaptic Plasticity

$2,2.2,45.5, \mathrm{P} 3$

$\mathrm{P} 52, \mathrm{P} 67, \mathrm{P} 182, \mathrm{P} 311$,

P462, P609, P621

synaptic pruning

P114

Synchrony

.26 .3

synspsis

396

systemic inflammation ................................................................................... P64

systems neurobiology ............................................................................ P494

systems neuroscience ........................................................................... 25

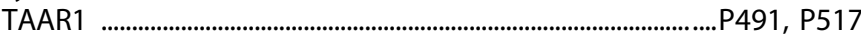

tactile mismatch ............................................................................ 2.4 
tandem repeat variation

P521

Target engagement

P507

targeted cognitive training

P562, P827

task fMRI

P455

Taste P680

tau

P409

Technology

P555

telemedicine

P556

temperament

44.3

temperature

P409

Test-Retest Reliability

P476

thalamo-cortical connectivity

P564

Thalamo-cortical Interactions

Thalamus

P537

45.2, 54.3, P24,

P260, P393, P784

P522

Thalamus - Reticular nucleus

THC ............................................... P589, P620, P631, P651, P682, P755

the COVID-19 pandemic ..........................37.5, P81, P272, P678, P816

theory of mind ............................................................................................. P527

therapeutic target ......................................................................................... P93

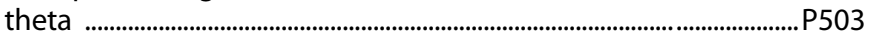

theta band oscillatory measures ……………………......................P470

Theta-burst stimulation

$50,50.4, P 232$

Threat of Shock

P260, P786

thyroid hormone ........................................................................................ 10

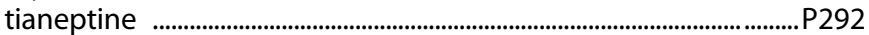

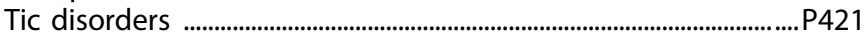

time-frequency ................................................................P436, P470, P669

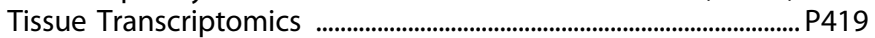

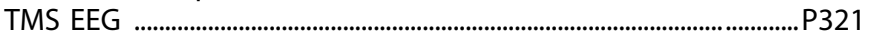

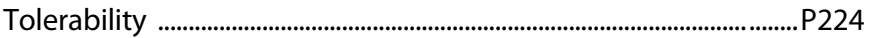

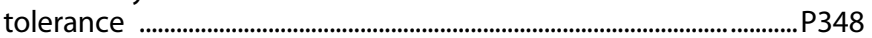

Toll-Like receptors (TLRs) ................................................31.3, P16, P592

top-down control .......................................................................................... P710

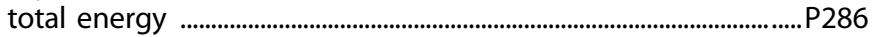

Tourette syndrome .....................................................P421, P423, P437

Trace amines-associated receptor ........................................................ 1 P705

T-RAD Study ..................................................................................................... P20

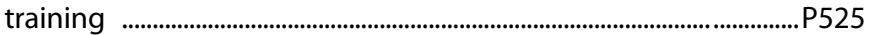

transcranial direct current stimulation ............................................. P652

transcranial magnetic stimulation (TMS) ....................... 50, 50.5, 55.2,

P250, P323, P392

transcranial photobiomodulation .......................................................P280

Transcriptome ........................................................28.4, P91, P636, P698

Transcriptomics ............................................... 29.3, 39, P123, P134, P360,

P657, P730, P794

transcutaneous auricular vagus

nerve stimulation (taVNS)

P326

Transdiagnostic

P433, P791, P804, P827

Transgender

Transgenic rats

translational

45.3, P711

Translational approaches to drug development ........................5, P311

Translational biomarker approaches

to drug development

P235

translational imaging

P137

Translational Neuroscience

$57,57.3$

transsynaptic

...P713

TRAP2 mice

P69

Trauma

... P84

Trauma exposure

27.1, 41.3, P34, P788

Traumatic Brain Injury (TBI)

P5, P24, P45, P425

Traumatic stress ................................................................................ 51, P783

Treatment Prediction

P749

treatment refractory

P480

treatment resistance $\ldots \ldots \ldots \ldots \ldots \ldots \ldots \ldots$
Treatment Resistant Depression

8.2, 8.3, 21.2, P231,

treatment targets

P273, P392

Treatment-resistance ............................................................................P350

Treatment-resistant depression

8.4, P245, P376

treatment-resistant late life depression

8.4, P 328

treatment-resistant schizophrenia

48.1, P566

Treatment-Response

P215, P227, P457

tricyclics

P262

T-type Calcium channel ..........................................................................P472

Two-photon ......................................................................................................P337

Two-photon calcium imaging ................................................ 45.4, P785

Type-2 Diabetes ........................................................................................ P53

Ubiquitin Proteasome System ...........................................................P449

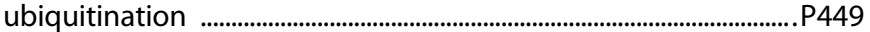

ultrasonic vocalization ................................................................................... P157

Uncinate Fasciculus ..................................................................................54.3

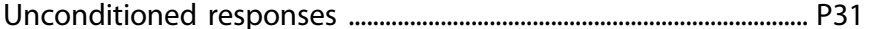

underrepresented ................................................................................................... 59

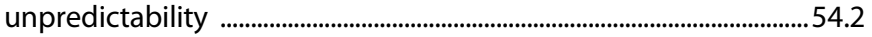

valence

P267, P337, P655

validity

..P524

Value-based Decision-Making

P738, P787, P792

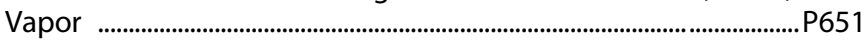

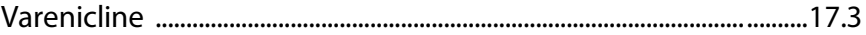

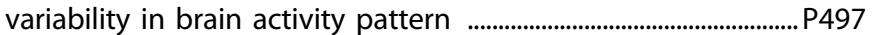

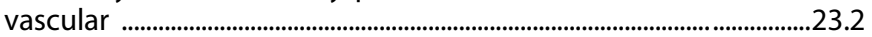

Vasopressin 1a receptor antagonist .................................................P154

ventral attention ........................................................................................... P28

ventral hippocampus ......................................... P50, P269, P489, P626

Ventral Pallidum ……………………….............. P73, P256, P615, P673

Ventral Striatum ..........................................................................P408, P571

Ventral tegmental area (VTA) .............................29.5, 57.5, P594, P689

ventral visual stream ..................................................................................P439

ventrolateral prefrontal cortex ................................................... 35, P270

ventromedial prefrontal cortex .........................................................P788

veterans ............................................................................ 17.1, P34, P477

VGluT2 neurons .......................................................................................... P427

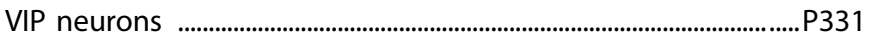

virtual reality ...............................................................................P562, P816

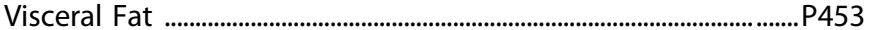

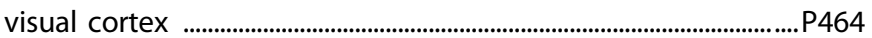

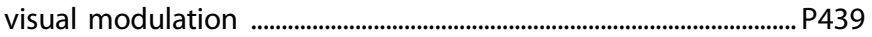

visual perception .................................................................................P483

visuospatial working memory ............................................................P531

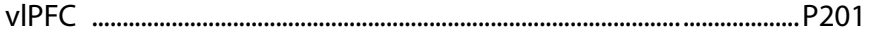

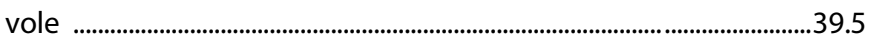

voltage indicator ............................................................................30, 30.2

Voxel-Based Morphometry (VBM) ........................................ 48.3, P156

VPAC2 receptor antagonist ............................................................... P553

vulnerability and resilience ......................................................................37.5

Vulnerability traits .....................................................................................P153

waist circumference .............................................................................. P335

White Matter

$14.1,27.2, \mathrm{P} 111$

White Matter Development .................................................................P107

white matter integrity ......................................................................... P586

white matter microstructure ................................................................... P85

WHO risk levels ...................................................................................... P610

Whole exome sequencing ............................................................. 48, P146

whole genome sequence ................................................................. P521

whole-genome ................................................................ 48.1, P566, P575

williams syndrome .................................................................... P147

withdrawal ................................................................................... P730, P759

Within-pair concordance .........................................................................26.2

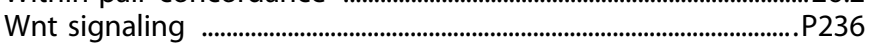

women ......................................................................................................... P48 
women's health

P76, P707

Women's Mental Health

$\mathrm{P} 18, \mathrm{P} 820$

working memory

working memory fMRI

6.5, P568, P620, P712

World Trade Center responders

Worry young adults

P332

youth

P133

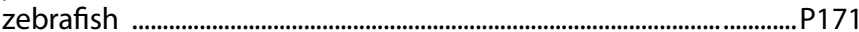

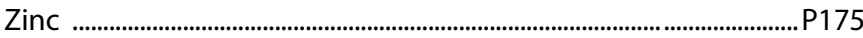

zuranolone ..........................................................P2222, P223, P224, P271 